\title{
Sweet Pepper Quality Maintenance: Impact of Hot Water and Chitosan
}

\author{
Neama M. Hussein", M M F. Abd Allah ${ }^{* *}$, A. Abou E-Yazied ${ }^{* *}$ \\ and Rawia E Ibrahim* \\ *Postharvest and Handling of Vegetable Department, \\ Horticulture Research Institute, Agricultural Research Centre \\ and ${ }^{* *}$ Horticulture Department, Faculty of Agriculture, Ain \\ Shams University, Shoubra El-Kheima, Cairo, Egypt.
}

\begin{abstract}
GWEET PEPPERS (Capsicum annuum $L$. testy red $\mathrm{F}_{1}$, hy brid) were grown at Ismailia Governorate during the two successive seasons of 2011/2012 and 2012-2013. Marketable fruits (3/4 red color) were used to study the effect of hot water at 25,45 and $55^{\circ} \mathrm{C}$ and Chitosan $(0,0.5$ and $1 \%$ ) treatments on the postharvest appearance, compositional quality as well as reducing chilling injury symptoms. Samples were stored at refrigerated storage $\left(5\right.$ or $8^{\circ} \mathrm{C}$ and $\left.90-95 \% \mathrm{RH}\right)$ for 28 days and were monitored weekly.Pepper fruits stored at $8^{\circ} \mathrm{C}$ did not show any pitting and seed darkening during all the storage periods, whereas this symptoms were noticed in fruits stored at $5^{\circ} \mathrm{C}$ after 14 days and became pronounced as the storage period extended. All tested treatments were effective in reducing weight loss, decay, shriveling, texture loss and chilling injury comp ared with untreated fruit during cold storage. Sweet peppers treated with hot water at $45^{\circ} \mathrm{C}$ and chitosan at 0.5 or $1 \%$ did not develop any chilling injury sy mptoms (expressed as pitting, caly $\mathrm{x}$ and seed darkening) during 21 days of storage at $5^{\circ} \mathrm{C}$, but after 28 days of storage traces of these symptoms were detected. Moreover the previous treatment maintained fruit texture, total carotenoids content and reduced decay incidence throughout all the storage periods. Therefore, hot water at $45^{\circ} \mathrm{C}$ and chitosan at 0.5 or $1 \%$ are promising treatments for reducing chilling injury and maintaining quality of sweet peppers during 28 days of storage at $5^{\circ} \mathrm{C}$ and $90-95 \mathrm{RH} \%$.
\end{abstract}

Keywards: (Capsicum annuum L.), Temperature, Hot water, Chitosan, Storage, Quality, Chilling injury.

Sweet bell pepper is one of the most important and popular vegetable crops grown for both local consumption and export. Bell peppers contains impressive list of plant nutrient that are found to have disease preventing and health promoting properties. (Howard et al., 2000). Major physiological and biochemical changes occur in pepper after harvest due to metabolic activities, which result in quality and nutritional value degradation as well as hastening senescence and decay. Mois ture los s results not only in appreciable weight loss but also in less attractive fruit due to poorer texture and wilted tissues, which lower the fruit quality. (Hardenburg et al., 1990 and Kader, 2002). Cooling is the primary mean of maintaining sweet pepper quality, where it 
lowers metabolism and decreases water loss, thus it prolong shelf life (Hardenburg et al., 1990, Kader, 2002 and Bayoumi, 2008). Sweet pepper fruit, being of tropical origin, are susceptible to chilling injury, if held at temperature lower than $7^{\circ} \mathrm{C}$ for more than 3 days (Kader, 2002 and Falliket et al., 2009). Lower temperature also accelerates incidence of surface mold and decay (Kader, 2002 and Lim et al., 2007). Pepper optimal storage conditions are a moderate temperature and a high humidity (95-98\%) so as to avoid chilling injury. At 7 to $10^{\circ} \mathrm{C}$ sound healthy peppers can be held for up to 3 weeks, depending on cultivar, state of maturity when picked, and post-harvest treatment used (Kader, 2002). Postharvest heat treatments were used to minimize moisture loss and decay(Maalekuu et al., 2003), maintain fruit firmness (Fallik et al., 1999 and Abdalla, 2013), retard fruit wilting and maintain fruit appearance (Maalekuu et al., 2005), inhibit color development, extend shelf life (Gonzalez-Aguilar et al., 1999) and reduce chilling injury (CI) symptoms (Ilic et al., 2012). Heat treatments offer an ample potential for maintaining fruit quality, which affect fruit tolerance to lower temperature and hence reduce the development of CI symptoms of sweet pepper during cold storage Bar-Yosef et al., 2009 and Fallik et $a l ., 2009)$.Another important technique that was not investigated intensively, but found to have a remarkable effect on maintaining quality and reducing CI symptoms is chitosan. Chitosan is a commercial product, It includes chitosan 90-95\%. (2Amino-2-deoxy-beta-D-glucosamine) EL-Badawy (2014). Chitosan treatment has been shown to be a us eful tool for enhancing quality maintenance, by retarding water loss, fruit softening and delay of ripening (Dong et al., 2004). Chitosan treatment reduce decay incidence (Raymond et al., 2012), inhibits fruit respiration (Xing et al., 2011),maintains color, extends the shelf life (Fard et al., 2010) and reduces CI symptoms in fruits(MengMeng et al., 2012). The effectiveness of chitos an and hot water treatments, alone or in combination to maintain quality and control of storage decay of sweet cherries was investigated by Chiloo and Asghari (2011) who found that a combined treatment of $0.5 \%$ chitos an and hot water at $50^{\circ} \mathrm{C}$ for 5 and $10 \mathrm{~min}$ was the best in controlling decay after 30 days of storage. The present study aims at investigating the effect of hot water and chitosan treatments on the maintenance of pepper postharvest quality, as well as investigating their impact on reducing CI incidence.

\section{Materials and Methods}

Sweet pepper (capsicum annum L.) hybrid testy were grown under plastic house conditions in private farm at Ismailia Governorate. Seeds were sown in the nursery on $8^{\text {th }}$ and $14^{\text {th }}$ of September in 2011 and 2012 seasons, respectively, and seedling were transplanted on $24^{\text {th }}$ of October in both seasons. The growing plants were subjected to regular inter cultural practices as well as diseases and pest control programs recommended by the Ministry of Agriculture for sweet pepper production. Storage experiment was carried out during the two successive seasons at in the laboratory of Vegetable Handling Research Department., at Giza Governorate. Sweet peppers were harvested at 3/4 red color stage and transferred directly to the postharvest laboratory. The marketable and uniform fruit were randomly dipped in hot water at $25^{\circ} \mathrm{C}$ tap water, (control), $45^{\circ} \mathrm{C}$ and

Egypt. J. Hort. Vol. 42, No.1 (2015) 
$55^{\circ} \mathrm{C}$ for 3 mints each. Then the samples were allowed to dry. Hot water treated peppers were sprayed with chitosan at $0.5 \%$ and $1 \%(5 \mathrm{~g}$ and $10 \mathrm{~g} /$ /iter $)$ or with tap water (control). Samples of each treatment were randomly packed in polystyrene trays, labeled and weighted. Treatments were arranged in a completely randomized design with three replicates (trays), each contained three fruit. All treatments were stored at $5^{\circ} \mathrm{Cor} 8^{\circ} \mathrm{C}$ and $90-95 \% \mathrm{RH}$ for $1,2,3$ or 4 weeks, respectively. All pepper treatments of the two seasons were devoted to the following physical and chemical analyses before and after storage. Pepper fruit were transferred from cold storage chambers to room temperature $\left(25^{\circ} \mathrm{C}\right)$. CI symptoms were evaluated after 5 hours from transferring peppers to room temperature. The degree of CI was judged by estimating the surface pitting area, calyx and seed darkening.

Weight loss was estimated according to the following equation:

Weight loss $\%=\frac{\text { Initial weight of fruits }- \text { weight of fruits at sampling date }}{\text { Initial weight loss }} \times 100$

Decay was measured on a scale of $1=$ none, $2=$ slight, $3=$ moderate, $4=$ severe, 5= extreme. (Ris se \& Miller, 1986).

Gloss: $5=$ none, $4=$ slight, $3=$ moderate, $2=$ severe, $1=$ extreme.

Shriveling: $1=$ none, $2=$ slight, $3=$ moderate, $4=$ severe, $5=$ extreme. (Ris se \& Miller, 1986).

Fruit texture was measured using fruit texture analyser Model GS-, serial No FTA2 expressed as $\mathrm{Lb} /$ inch $^{2}$

Surface pitting area was determined using the following system:

Score $1=$ no abnormality, $2=$ trace, $3=$ slight, $4=$ moderate and $5=$ severe as described by Wang and Qi (1997).

Calyx darkening was estimated according to the following Score: a scale of 1 to 5 , with $1=$ no abnormality, $2=$ trace, $3=$ slight, $4=$ moderate and $5=$ severe as described by Wang and Qi (1997).

Seed darkening was estimated according to the following a scale of 1 to 5 , with $1=$ no abnormality, $2=$ trace, $3=$ slight, $4=$ moderate and $5=$ severe as described by Wang and Qi (1997).

Total carotenoids content $(\mathrm{mg} / 100 \mathrm{~g}$ fresh weight) was determined according to A.O.A.C. (1990).

Total soluble solids percentage (TSS) \% was measured from the fresh materials using PR-101 digital refractometer

Statistical analysis

Data were subjected to statistical analy sis for calculation of means, variance and stander error according to MSTATC software. Mean separations were estimated by calculating LSD value at 5\% level according to Snedecor and Cochran (1980). 


\section{Results and Discution}

Data presented in Table 1 show the influence of hot water treatments, chitosan and storage temperature on weight loss of sweet pepper fruits during storage in the two seasons (2011/2012 and 2012/2013). In general, stored pepper showed significant weight loss as the storage period extended, the highest weight loss values were obtained at the end of storage. Similar results were reported by Fallik et al. (1999). There were significant differences between hot water, chitosan treatments and storage temperature in weight loss in every storage period. Moreover, all treatments retained their weight during storage as compared with untreated control. After 28 days of storage, sweet peppers treated with hot water at $45^{\circ} \mathrm{C}+$ chitosan at $0.5 \%$ and stored at $5^{\circ} \mathrm{C}$ had the lowest weight loss $(4.52 \%)$ followed by hot water at $45^{\circ} \mathrm{C}+$ chitosan at $1 \%$ and stored at $5^{\circ} \mathrm{C}(4.73 \%)$ with significant differences between them. However, the highest weight loss $6.41 \%$ was obtained from untreated control either for hot water or chitosan treatments and stored at $8^{\circ} \mathrm{C}$ in the first season. In general, in all treatments and control, sweet peppers stored at $5^{\circ} \mathrm{C}$ exhibited the least loss in weight, while, peppers stored at $8^{\circ} \mathrm{C}$ had the highest. These results in agreement with those obtained by Djioua et al. (2010) in fresh-cut mangoes and Chailoo and Asghari (2011) in sweet cherry. Weight loss is natural consequence of the catabolism of horticultural products, and attributed to respiration and other senescence related metabolic processes during storage (Watada and Qi, 1999). The favorable effect of chitosan treatment in reducing weight loss may be due to the formation of thin layer covering the fruit, which prevents moisture loss, reduces gas exchange and subsequently inhibits metabolic activities, resulting in lowering weight loss (Raymond et al., 2012).

The results in Table 2 demonstrate the influence of hot water, chitosan and storage temperature on decay of s weet peppers during storage in the two seasons. No decay was observed in all treatments in peppers stored for 7 or 14 days. However, after 28 days of storage there were significant differences between all treatments and control. Also, no decay was observed in sweet peppers treated with hot water at $45^{\circ} \mathrm{C}$ + chitos an at 0.5 or $1 \%$ and stored at $5^{\circ} \mathrm{C}$ or $8^{\circ} \mathrm{C}$, and hot water at $55^{\circ} \mathrm{C}+$ chitosan at $0.5 \%$ and stored at $8^{\circ} \mathrm{C}$, while untreated control stored at $5^{\circ} \mathrm{C}$ had the highest incidence of decay.These results agree with those of Chailoo and Asghari (2011) who found that chitosan and hot water treatments were effective in reducing decay of sweet cherries during storage. Also, Djioua et al. (2010) reported that the antibacterial action of chitosan coating of $0.25 \%$ was more effective when applied with heat treatment at $50^{\circ} \mathrm{C}$ for $30 \mathrm{~min}$. to reduce decay of mangoes during storage.Chitosan coating and hot water treatments induce resistance in the host by increasing chitinase and b-1, 3 glucanase in strawberries (Fajardo et al ., 1998) and phenylanine ammonia - lyase (PAL) activity in table grapes (Romanazzi et al., 2002). Moreover, they increase the level of 6 - methoxy - mullein, the principatphytonlexin of carrots (Reddy et al., 1999). Physical treatments elicit responses in harvested commodities, increasing enzymatic activity (e-g .PAL, and peroxidase) related to host resistance against pathogens (Wilson et al., 1994).

Egypt. J. Hort. Vol. 42, No.1 (2015) 
TABLE 1. Effect of hot water, chitosan treatments and storage temperature on weight loss $\%$ of sweet pepper fruits (Testy red $F_{1}$ Hybrid) during cold storage period in 2011 / 2012 and 2012 / 2013 seasons.

\begin{tabular}{|c|c|c|c|c|c|c|c|c|c|c|c|}
\hline \multirow{2}{*}{\multicolumn{2}{|c|}{ Treatments }} & \multicolumn{10}{|c|}{ Storage period (days) } \\
\hline & & \multicolumn{2}{|c|}{$\mathbf{0}$} & \multicolumn{2}{|c|}{7} & \multicolumn{2}{|c|}{14} & \multicolumn{2}{|r|}{21} & \multicolumn{2}{|c|}{28} \\
\hline & & \multicolumn{10}{|c|}{$2011 / 2012$} \\
\hline 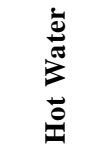 & 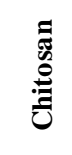 & $5^{\circ} \mathrm{C}$ & $8^{\circ} \mathrm{C}$ & $5^{\circ} \mathrm{C}$ & $8^{\circ} \mathrm{C}$ & $5^{\circ} \mathrm{C}$ & $8^{\circ} \mathrm{C}$ & $5^{\circ} \mathrm{C}$ & $8^{\circ} \mathrm{C}$ & $5^{\circ} \mathrm{C}$ & $8^{\circ} \mathrm{C}$ \\
\hline \multirow{3}{*}{$\begin{array}{c}\text { Control } \\
25^{\circ} \mathrm{c}\end{array}$} & 0 & $0.00 \mathrm{a}$ & $0.00 \mathrm{a}$ & $0.69 \mathrm{c}$ & $1.30 \mathrm{a}$ & $1.86 \mathrm{~d}$ & $2.62 \mathrm{a}$ & $3.94 \mathrm{~g}$ & $\mathrm{~g} 4.92 \mathrm{a}$ & $5.24 \mathrm{~g}$ & $6.41 \mathrm{a}$ \\
\hline & $0.5 \%$ & $0.00 \mathrm{a}$ & $0.00 \mathrm{a}$ & $0.55 \mathrm{~cd}$ & $1.20 \mathrm{ab}$ & $1.49 \mathrm{~g}$ & $2.51 \mathrm{ab}$ & $3.61 \mathrm{i}$ & i $4.74 \mathrm{bc}$ & $5.03 \mathrm{ij}$ & $\mathrm{j} \mid 6.00 \mathrm{c}$ \\
\hline & $1 \%$ & $0.00 \mathrm{a}$ & $0.00 \mathrm{a}$ & $0.57 \mathrm{~cd}$ & $1.23 \mathrm{ab}$ & $1.80 \mathrm{de}$ & $2.50 \mathrm{ab}$ & $3.74 \mathrm{~h}$ & $\mathrm{~h} 4.87 \mathrm{ab}$ & $5.11 \mathrm{~h}$ & $6.20 \mathrm{~b}$ \\
\hline \multirow{3}{*}{$45^{\circ} \mathrm{C}$} & 0 & $0.00 \mathrm{a}$ & $0.00 \mathrm{a}$ & $0.50 \mathrm{~cd}$ & $1.10 \mathrm{ab}$ & $1.72 \mathrm{dg}$ & $2.42 \mathrm{a}-\mathrm{c}$ & c $3.41 \mathrm{j}$ & j $4.61 \mathrm{e}$ & $4.91 \mathrm{k}$ & $5.84 \mathrm{~d}$ \\
\hline & $0.5 \%$ & $0.00 \mathrm{a}$ & $0.00 \mathrm{a}$ & $0.40 \quad \mathrm{a}$ & $1.00 \mathrm{~b}$ & $1.54 \mathrm{fg}$ & $2.21 \mathrm{c}$ & 3.201 & $14.42 \mathrm{f}$ & $4.52 \mathrm{n}$ & $5.52 \mathrm{f}$ \\
\hline & $1 \%$ & $0.00 \mathrm{a}$ & $0.00 \mathrm{a}$ & $0.42 \mathrm{~d}$ & $1.06 \mathrm{~b}$ & $1.62 \mathrm{e}-\mathrm{g}$ & $2.30 \mathrm{bc}$ & $3.30 \mathrm{k}$ & $\begin{array}{l}\mathrm{k} \\
\mathrm{k}\end{array}$ & $4.73 \mathrm{~m}$ & $5.64 \mathrm{e}$ \\
\hline \multirow{3}{*}{$55^{\circ} \mathrm{C}$} & 0 & $0.00 \mathrm{a}$ & $0.00 \mathrm{a}$ & $0.60 \mathrm{~cd}$ & $1.20 \mathrm{ab}$ & $1.81 \mathrm{de}$ & $2.50 \mathrm{ab}$ & $3.61 \mathrm{i}$ & $\mathrm{i} 4.75 \mathrm{~cd}$ & $5.10 \mathrm{hi}$ & ii $6.06 \mathrm{c}$ \\
\hline & $0.5 \%$ & $0.00 \mathrm{a}$ & $0.00 \mathrm{a}$ & $0.50 \mathrm{~cd}$ & $1.10 \mathrm{ab}$ & $1.62 \mathrm{e}-\mathrm{g}$ & $2.30 \mathrm{bc}$ & $3.42 \mathrm{j}$ & $\mathrm{j} \mid 4.62 \mathrm{e}$ & 4.821 & $5.84 \mathrm{~d}$ \\
\hline & $1 \%$ & $0.00 \mathrm{a}$ & $0.00 \mathrm{a}$ & $0.54 \mathrm{~cd}$ & $1.16 \mathrm{ab}$ & $1.74 \mathrm{dtf}$ & $2.40 \mathrm{a}-\mathrm{c}$ & $3.54 \mathrm{i}$ & i $4.70 \mathrm{~d}$ & $5.00 \mathrm{j}$ & $5.71 \mathrm{e}$ \\
\hline \multicolumn{12}{|c|}{$2012 / 2013$} \\
\hline \multirow{3}{*}{$\begin{array}{c}\text { Control } \\
25^{\circ} \mathrm{c}\end{array}$} & 0 & $0.00 \mathrm{a}$ & $0.00 \mathrm{a}$ & $0.87 \mathrm{~cd}$ & $1.48 \mathrm{a}$ & $2.76 \mathrm{de}$ & $3.36 \mathrm{a}$ & $4.78 \mathrm{e}$ & $6.00 \mathrm{a}$ & $5.94 \mathrm{f}$ & $=7.61 \mathrm{a}$ \\
\hline & $0.5 \%$ & $0.00 \mathrm{a}$ & $0.00 \mathrm{a}$ & $0.55 \mathrm{gi}$ & $0.88 \mathrm{c}$ & $1.93 \mathrm{i}$ & $2.69 \mathrm{e}$ & $3.83 \mathrm{j}$ & $\mathrm{j} \mid 4.54 \mathrm{fg}$ & $5.17 \mathrm{jk}$ & \begin{tabular}{l|l}
$k$ & $6.13 \mathrm{e}$
\end{tabular} \\
\hline & $1 \%$ & $0.00 \mathrm{a}$ & $0.00 \mathrm{a}$ & $0.69 \mathrm{e}$ & $1.05 \mathrm{~b}$ & $2.43 \mathrm{~h}$ & $3.11 \mathrm{~b}$ & $4.50 \mathrm{~g}$ & $\mathrm{~g} \mid 4.91 \mathrm{~d}$ & $5.66 \mathrm{~h}$ & $6.32 \mathrm{~d}$ \\
\hline \multirow{3}{*}{$45^{\circ} \mathrm{C}$} & 0 & $0.00 \mathrm{a}$ & $0.00 \mathrm{a}$ & $0.42 \mathrm{j}-1$ & $10.58 \mathrm{f}-\mathrm{h}$ & $2.51 \mathrm{~g}$ & $3.12 \mathrm{~b}$ & $4.49 \mathrm{~g}$ & \begin{tabular}{l|l}
$\mathrm{g}$ & $5.00 \mathrm{c}$
\end{tabular} & $5.22 \mathrm{j}$ & $6.52 \mathrm{c}$ \\
\hline & $0.5 \%$ & $0.00 \mathrm{a}$ & $0.00 \mathrm{a}$ & 0.361 & $0.48 \mathrm{ij}$ & $1.68 \mathrm{~lm}$ & $2.78 \mathrm{~d}$ & 2.641 & $13.82 \mathrm{j}$ & $4.28 \mathrm{o}$ & $5.14 \mathrm{k}$ \\
\hline & $1 \%$ & $0.00 \mathrm{a}$ & $0.00 \mathrm{a}$ & $0.40 \mathrm{kl}$ & $0.53 \mathrm{hi}$ & $1.79 \mathrm{jk}$ & $2.90 \mathrm{c}$ & $.20 \mathrm{~m}$ & $\mathrm{~m} 4.10 \mathrm{~h}$ & $4.57 \mathrm{n}$ & $5.32 \mathrm{i}$ \\
\hline \multirow{3}{*}{$55^{\circ} \mathrm{C}$} & 0 & $0.00 \mathrm{a}$ & $0.00 \mathrm{a}$ & $0.77 \mathrm{~d}$ & $1.00 \mathrm{~b}$ & $2.61 \mathrm{f}$ & $2.83 \mathrm{~cd}$ & $4.60 \mathrm{f}$ & $\begin{array}{l}\mathrm{f} \\
5.83 \mathrm{~b}\end{array}$ & $5.64 \mathrm{~h}$ & $6.94 \mathrm{~b}$ \\
\hline & $0.5 \%$ & $0.00 \mathrm{a}$ & $0.00 \mathrm{a}$ & $0.45 \mathrm{jk}$ & $0.62 \mathrm{e}-\mathrm{g}$ & $1.86 \mathrm{ij}$ & $1.75 \mathrm{kl}$ & $3.54 \mathrm{k}$ & $\mathrm{k} / .10 \mathrm{~h}$ & $4.76 \mathrm{~m}$ & $\mathrm{n} 5.78 \mathrm{~g}$ \\
\hline & $1 \%$ & $0.00 \mathrm{a}$ & $0.00 \mathrm{a}$ & 0.64 ef & f $0.66 \mathrm{e}$ & $1.59 \mathrm{n}$ & $1.61 \mathrm{mn}$ & $3.92 \mathrm{i}$ & $\mathrm{i} \mid 4.61 \mathrm{f}$ & 5.001 & $5.95 \mathrm{f}$ \\
\hline
\end{tabular}


TABLE 2. Effect of hot water, chitosan treatments and storage temperature on decay (score)* of sweet red pepper fruits (Testy red $\mathbf{F}_{1}$ Hybrid) during cold storage in 2011/2012 and 2012/2013 seasons.

\begin{tabular}{|c|c|c|c|c|c|c|c|c|c|c|c|}
\hline \multirow{3}{*}{\multicolumn{2}{|c|}{ Treatments }} & \multicolumn{10}{|c|}{ Storage period (days) } \\
\hline & & \multicolumn{2}{|c|}{$\mathbf{0}$} & \multicolumn{2}{|c|}{7} & \multicolumn{2}{|c|}{14} & \multicolumn{2}{|c|}{21} & \multicolumn{2}{|c|}{28} \\
\hline & & \multicolumn{10}{|c|}{$2011 / 2012$} \\
\hline 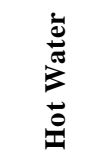 & 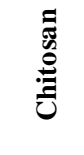 & $5^{\circ} \mathrm{C}$ & $8^{\circ} \mathrm{C}$ & $5^{\circ} \mathrm{C}$ & $8^{\circ} \mathrm{C}$ & $5^{\circ} \mathrm{C}$ & $8^{\circ} \mathrm{C}$ & $5^{\circ} \mathrm{C}$ & $8^{\circ} \mathrm{C}$ & $5^{\circ} \mathrm{C}$ & $8^{\circ} \mathrm{C}$ \\
\hline \multirow{3}{*}{$\begin{array}{l}\text { Control } \\
25^{\circ} \mathrm{c}\end{array}$} & 0 & $1.00 \mathrm{a}$ & $1.00 \mathrm{a}$ & $1.00 \mathrm{a}$ & $1.00 \mathrm{a}$ & $1.00 \mathrm{a}$ & $1.00 \mathrm{a}$ & $2.33 \mathrm{a}$ & $1.00 \mathrm{~d}$ & $3.66 \mathrm{a}$ & $2.00 \mathrm{~b}$ \\
\hline & $0.5 \%$ & $1.00 \mathrm{a}$ & $1.00 \mathrm{a}$ & $1.00 \mathrm{a}$ & $1.00 \mathrm{a}$ & $1.00 \mathrm{a}$ & $1.00 \mathrm{a}$ & $1.66 \mathrm{~b}$ & $1.00 \mathrm{~d}$ & $2.00 \mathrm{~b}$ & $1.33 \mathrm{~d}$ \\
\hline & $1 \%$ & $1.00 \mathrm{a}$ & $1.00 \mathrm{a}$ & $1.00 \mathrm{a}$ & $1.00 \mathrm{a}$ & $1.00 \mathrm{a}$ & $1.00 \mathrm{a}$ & $1.66 \mathrm{~b}$ & $1.00 \mathrm{~d}$ & $2.00 \mathrm{~b}$ & $1.33 \mathrm{~d}$ \\
\hline \multirow{3}{*}{$45^{\circ} \mathrm{C}$} & 0 & $1.00 \mathrm{a}$ & $1.00 \mathrm{a}$ & $1.00 \mathrm{a}$ & $1.00 \mathrm{a}$ & $1.00 \mathrm{a}$ & $1.00 \mathrm{a}$ & $1.00 \mathrm{~d}$ & $1.00 \mathrm{~d}$ & $1.66 \mathrm{c}$ & $1.66 \mathrm{c}$ \\
\hline & $0.5 \%$ & $1.00 \mathrm{a}$ & $1.00 \mathrm{a}$ & $1.00 \mathrm{a}$ & $1.00 \mathrm{a}$ & $1.00 \mathrm{a}$ & $1.00 \mathrm{a}$ & $1.00 \mathrm{~d}$ & $1.00 \mathrm{~d}$ & $1.00 \mathrm{e}$ & $1.00 \mathrm{e}$ \\
\hline & $1 \%$ & $1.00 \mathrm{a}$ & $1.00 \mathrm{a}$ & $1.00 \mathrm{a}$ & $1.00 \mathrm{a}$ & $1.00 \mathrm{a}$ & $1.00 \mathrm{a}$ & $1.00 \mathrm{~d}$ & $1.00 \mathrm{~d}$ & $1.00 \mathrm{e}$ & $1.00 \mathrm{e}$ \\
\hline \multirow{3}{*}{$55^{\circ} \mathrm{C}$} & 0 & $1.00 \mathrm{a}$ & $1.00 \mathrm{a}$ & $1.00 \mathrm{a}$ & $1.00 \mathrm{a}$ & $1.00 \mathrm{a}$ & $1.00 \mathrm{a}$ & $1.33 \mathrm{c}$ & $1.00 \mathrm{~d}$ & $2.00 \mathrm{~b}$ & $1.66 \mathrm{c}$ \\
\hline & $0.5 \%$ & $1.00 \mathrm{a}$ & $1.00 \mathrm{a}$ & $1.00 \mathrm{a}$ & $1.00 \mathrm{a}$ & $1.00 \mathrm{a}$ & $1.00 \mathrm{a}$ & $1.00 \mathrm{~d}$ & $1.00 \mathrm{~d}$ & $1.33 \mathrm{~d}$ & $1.00 \mathrm{e}$ \\
\hline & $1 \%$ & $1.00 \mathrm{a}$ & $1.00 \mathrm{a}$ & $1.00 \mathrm{a}$ & $1.00 \mathrm{a}$ & $1.00 \mathrm{a}$ & $1.00 \mathrm{a}$ & $1.00 \mathrm{~d}$ & $1.00 \mathrm{~d}$ & $1.66 \mathrm{c}$ & $1.33 \mathrm{~d}$ \\
\hline \multicolumn{12}{|c|}{$2012 / 2013$} \\
\hline \multirow{3}{*}{$\begin{array}{l}\text { Control } \\
25^{\circ} \mathrm{c}\end{array}$} & 0 & $1.00 \mathrm{a}$ & $1.00 \mathrm{a}$ & $1.00 \mathrm{a}$ & $1.00 \mathrm{a}$ & $1.00 \mathrm{a}$ & $1.00 \mathrm{a}$ & $2.66 \mathrm{a}$ & $1.00 \mathrm{~d}$ & $4.00 \mathrm{a}$ & $2.66 \mathrm{~b}$ \\
\hline & $0.5 \%$ & $1.00 \mathrm{a}$ & $1.00 \mathrm{a}$ & $1.00 \mathrm{a}$ & $1.00 \mathrm{a}$ & $1.00 \mathrm{a}$ & $1.00 \mathrm{a}$ & $1.33 \mathrm{c}$ & $1.00 \mathrm{~d}$ & $2.33 \mathrm{c}$ & $1.66 \mathrm{e}$ \\
\hline & $1 \%$ & $1.00 \mathrm{a}$ & $1.00 \mathrm{a}$ & $1.00 \mathrm{a}$ & $1.00 \mathrm{a}$ & $1.00 \mathrm{a}$ & $1.00 \mathrm{a}$ & $1.66 \mathrm{~b}$ & $1.00 \mathrm{~d}$ & $2.33 \mathrm{c}$ & $1.66 \mathrm{e}$ \\
\hline \multirow{3}{*}{$45^{\circ} \mathrm{C}$} & 0 & $1.00 \mathrm{a}$ & $1.00 \mathrm{a}$ & $1.00 \mathrm{a}$ & $1.00 \mathrm{a}$ & $1.00 \mathrm{a}$ & $1.00 \mathrm{a}$ & $1.33 \mathrm{c}$ & $1.00 \mathrm{~d}$ & $1.66 \mathrm{e}$ & $1.33 \mathrm{f}$ \\
\hline & $0.5 \%$ & $1.00 \mathrm{a}$ & $1.00 \mathrm{a}$ & $1.00 \mathrm{a}$ & $1.00 \mathrm{a}$ & $1.00 \mathrm{a}$ & $1.00 \mathrm{a}$ & $1.00 \mathrm{~d}$ & $1.00 \mathrm{~d}$ & $1.00 \mathrm{~g}$ & $1.00 \mathrm{~g}$ \\
\hline & $1 \%$ & $1.00 \mathrm{a}$ & $1.00 \mathrm{a}$ & $1.00 \mathrm{a}$ & $1.00 \mathrm{a}$ & $1.00 \mathrm{a}$ & $1.00 \mathrm{a}$ & $1.00 \mathrm{~d}$ & $1.00 \mathrm{~d}$ & $1.00 \mathrm{~g}$ & $1.00 \mathrm{~g}$ \\
\hline \multirow{3}{*}{$55^{\circ} \mathrm{C}$} & 0 & $1.00 \mathrm{a}$ & $1.00 \mathrm{a}$ & $1.00 \mathrm{a}$ & $1.00 \mathrm{a}$ & $1.00 \mathrm{a}$ & $1.00 \mathrm{a}$ & $1.66 \mathrm{~b}$ & $1.00 \mathrm{~d}$ & $2.33 \mathrm{c}$ & $2.00 \mathrm{~d}$ \\
\hline & $0.5 \%$ & $1.00 \mathrm{a}$ & $1.00 \mathrm{a}$ & $1.00 \mathrm{a}$ & $1.00 \mathrm{a}$ & $1.00 \mathrm{a}$ & $1.00 \mathrm{a}$ & $1.00 \mathrm{~d}$ & $1.00 \mathrm{~d}$ & $1.66 \mathrm{e}$ & $1.00 \mathrm{~g}$ \\
\hline & $1 \%$ & $1.00 \mathrm{a}$ & $1.00 \mathrm{a}$ & $1.00 \mathrm{a}$ & $1.00 \mathrm{a}$ & $1.00 \mathrm{a}$ & $1.00 \mathrm{a}$ & $1.00 \mathrm{~d}$ & $1.00 \mathrm{~d}$ & $1.66 \mathrm{e}$ & $1.66 \mathrm{e}$ \\
\hline
\end{tabular}

The influence of hot water, chitos an and storage temperature on gloss of sweet peppers during storage in the two seasons is illustrated in Table (3). Results proved that sweet peppers in all treatments did not exhibit any changes in their gloss among different treatments within 7 days of storage at 5 and $8^{\circ} \mathrm{C}$. However, after 28 days of storage there were significant differences between all treatments and untreated control in gloss. Sweet peppers treated with hot water at $45^{\circ} \mathrm{C}$ and treat ed with chitos an at the two concentrations and stored at 5 and $8^{\circ} \mathrm{C}$ showed the highest 
value of gloss, while untreated control stored at $5^{\circ} \mathrm{C}$ showed the lowest gloss . These results are agreement with Chailoo and Asghari (2011) on sweet cherry, In general, in all treatments and control sweet peppers stored at $8^{\circ} \mathrm{C}$ had the highest value of glos s scores, while those stored at $5^{\circ} \mathrm{C}$ gave the lowest ones. These results are in agreement with those obtained by Rodriguez et al. (2005) who found that hot water treatments were greatly beneficial in improving visual quality of peppers. Raymond et al. (2012) reported that the cut peppers coated with chitos an at $1 \%$ and $2 \%$ had higher L color value during storage probably due to the added glossiness of the coating. On the other hand, the surface lightness of the control treatment clearly reduced after storage for 5 day.

TABLE 3. Effect of hot water, chitosan treatments and storage temperature on gloss (score)* of sweet red pepper fruits (Testy red $\mathrm{F}_{1}$ Hybrid) during cold storage in 2011/2012 and 2012/2013 seasons.

\begin{tabular}{|c|c|c|c|c|c|c|c|c|c|c|c|}
\hline \multirow{3}{*}{\multicolumn{2}{|c|}{ Treatments }} & \multicolumn{10}{|c|}{ Storage period (days) } \\
\hline & & \multicolumn{2}{|r|}{$\mathbf{0}$} & \multicolumn{2}{|c|}{7} & \multicolumn{2}{|c|}{14} & \multicolumn{2}{|c|}{21} & \multicolumn{2}{|c|}{28} \\
\hline & & \multicolumn{10}{|c|}{$2011 / 2012$} \\
\hline 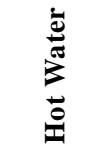 & 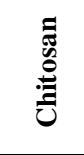 & $5^{\circ} \mathrm{C}$ & $8^{\circ} \mathrm{C}$ & $5^{\circ} \mathrm{C}$ & $8^{\circ} \mathrm{C}$ & $5^{\circ} \mathrm{C}$ & $8^{\circ} \mathrm{C}$ & $5^{\circ} \mathrm{C}$ & $8^{\circ} \mathrm{C}$ & $5^{\circ} \mathrm{C}$ & $8^{\circ} \mathrm{C}$ \\
\hline \multirow{3}{*}{$\begin{array}{c}\text { Control } \\
25^{\circ} \mathrm{C}\end{array}$} & 0 & $5.00 \mathrm{a}$ & $5.00 \mathrm{a}$ & $5.00 \mathrm{a}$ & $5.00 \mathrm{a}$ & $3.00 \mathrm{f}$ & $4.00 \mathrm{~d}$ & $2.00 \mathrm{f}$ & $3.00 \mathrm{e}$ & $1.00 \mathrm{~g}$ & $2.00 \mathrm{f}$ \\
\hline & $0.5 \%$ & $5.00 \mathrm{a}$ & $5.00 \mathrm{a}$ & $5.00 \mathrm{a}$ & $5.00 \mathrm{a}$ & $4.00 \mathrm{~d}$ & $4.67 \mathrm{~b}$ & $3.00 \mathrm{e}$ & $3.00 \mathrm{e}$ & $2.00 \mathrm{f}$ & $3.00 \mathrm{c}$ \\
\hline & $1 \%$ & $5.00 \mathrm{a}$ & $5.00 \mathrm{a}$ & $5.00 \mathrm{a}$ & $5.00 \mathrm{a}$ & $4.00 \mathrm{~d}$ & $4.67 \mathrm{~b}$ & $3.00 \mathrm{e}$ & $3.00 \mathrm{e}$ & $2.00 \mathrm{f}$ & $3.00 \mathrm{c}$ \\
\hline \multirow{3}{*}{$45^{\circ} \mathrm{C}$} & 0 & $5.00 \mathrm{a}$ & $5.00 \mathrm{a}$ & $5.00 \mathrm{a}$ & $5.00 \mathrm{a}$ & $4.00 \mathrm{~d}$ & $4.00 \mathrm{~d}$ & $3.00 \mathrm{e}$ & $3.33 \mathrm{~d}$ & $2.33 \mathrm{e}$ & $3.00 \mathrm{c}$ \\
\hline & $0.5 \%$ & $5.00 \mathrm{a}$ & $5.00 \mathrm{a}$ & $5.00 \mathrm{a}$ & $5.00 \mathrm{a}$ & $4.33 \mathrm{c}$ & $5.00 \mathrm{a}$ & $3.67 \mathrm{c}$ & $4.33 \mathrm{a}$ & $3.33 \mathrm{~b}$ & $3.67 \mathrm{a}$ \\
\hline & $1 \%$ & $5.00 \mathrm{a}$ & $5.00 \mathrm{a}$ & $5.00 \mathrm{a}$ & $5.00 \mathrm{a}$ & $4.33 \mathrm{c}$ & $5.00 \mathrm{a}$ & $3.67 \mathrm{c}$ & $4.00 \mathrm{~b}$ & $3.33 \mathrm{~b}$ & $3.67 \mathrm{a}$ \\
\hline \multirow{3}{*}{$55^{\circ} \mathrm{C}$} & 0 & $5.00 \mathrm{a}$ & $5.00 \mathrm{a}$ & $5.00 \mathrm{a}$ & $5.00 \mathrm{a}$ & $3.33 \mathrm{e}$ & $4.00 \mathrm{~d}$ & $3.00 \mathrm{e}$ & $3.33 \mathrm{~d}$ & $2.33 \mathrm{e}$ & $2.67 \mathrm{~d}$ \\
\hline & $0.5 \%$ & $5.00 \mathrm{a}$ & $5.00 \mathrm{a}$ & $5.00 \mathrm{a}$ & $5.00 \mathrm{a}$ & $4.00 \mathrm{~d}$ & $4.67 \mathrm{~b}$ & $3.33 \mathrm{~d}$ & $3.67 \mathrm{c}$ & $3.00 \mathrm{c}$ & $3.33 \mathrm{~b}$ \\
\hline & $1 \%$ & $5.00 \mathrm{a}$ & $5.00 \mathrm{a}$ & $5.00 \mathrm{a}$ & $5.00 \mathrm{a}$ & $4.00 \mathrm{~d}$ & $4.67 \mathrm{~b}$ & $3.33 \mathrm{~d}$ & $3.67 \mathrm{c}$ & $3.00 \mathrm{c}$ & $3.33 \mathrm{~b}$ \\
\hline \multicolumn{12}{|c|}{$2012 / 2013$} \\
\hline \multirow{3}{*}{$\begin{array}{c}\text { Control } \\
25^{\circ} \mathrm{C}\end{array}$} & 0 & $5.00 \mathrm{a}$ & $5.00 \mathrm{a}$ & $5.00 \mathrm{a}$ & $5.00 \mathrm{a}$ & $3.00 \mathrm{e}$ & $3.67 \mathrm{~d}$ & $2.00 \mathrm{f}$ & $3.00 \mathrm{e}$ & $1.00 \mathrm{~g}$ & $2.67 \mathrm{~d}$ \\
\hline & $0.5 \%$ & $5.00 \mathrm{a}$ & $5.00 \mathrm{a}$ & $5.00 \mathrm{a}$ & $5.00 \mathrm{a}$ & $3.67 \mathrm{~d}$ & $4.33 \mathrm{~b}$ & $3.00 \mathrm{e}$ & $3.67 \mathrm{cc}$ & $2.00 \mathrm{f}$ & $3.00 \mathrm{c}$ \\
\hline & $1 \%$ & $5.00 \mathrm{a}$ & $5.00 \mathrm{a}$ & $5.00 \mathrm{a}$ & $5.00 \mathrm{a}$ & $3.67 \mathrm{~d}$ & $4.33 \mathrm{~b}$ & $3.00 \mathrm{e}$ & $3.67 \mathrm{c}$ & $2.00 \mathrm{f}$ & $3.00 \mathrm{c}$ \\
\hline \multirow{3}{*}{$45^{\circ} \mathrm{C}$} & 0 & $5.00 \mathrm{a}$ & $5.00 \mathrm{a}$ & $5.00 \mathrm{a}$ & $5.00 \mathrm{a}$ & $3.67 \mathrm{~d}$ & $4.00 \mathrm{c}$ & $3.00 \mathrm{e}$ & $3.67 \mathrm{c}$ & $2.66 \mathrm{~d}$ & $3.00 \mathrm{c}$ \\
\hline & $0.5 \%$ & $5.00 \mathrm{a}$ & $5.00 \mathrm{a}$ & $5.00 \mathrm{a}$ & $5.00 \mathrm{a}$ & $4.00 \mathrm{c}$ & $5.00 \mathrm{a}$ & $3.67 \mathrm{c}$ & $4.67 \mathrm{a}$ & $3.33 \mathrm{~b}$ & $3.67 \mathrm{a}$ \\
\hline & $1 \%$ & $5.00 \mathrm{a}$ & $5.00 \mathrm{a}$ & $5.00 \mathrm{a}$ & $5.00 \mathrm{a}$ & $4.00 \mathrm{c}$ & $5.00 \mathrm{a}$ & $3.67 \mathrm{c}$ & $4.67 \mathrm{a}$ & $3.33 \mathrm{~b}$ & $3.67 \mathrm{a}$ \\
\hline \multirow{3}{*}{$55^{\circ} \mathrm{C}$} & 0 & $5.00 \mathrm{a}$ & $5.00 \mathrm{a}$ & $5.00 \mathrm{a}$ & $5.00 \mathrm{a}$ & $3.67 \mathrm{~d}$ & $4.00 \mathrm{c}$ & $3.00 \mathrm{e}$ & $3.33 \mathrm{~d}$ & $2.33 \mathrm{e}$ & $3.00 \mathrm{c}$ \\
\hline & $0.5 \%$ & $5.00 \mathrm{a}$ & $5.00 \mathrm{a}$ & $5.00 \mathrm{a}$ & $5.00 \mathrm{a}$ & $4.00 \mathrm{c}$ & $5.00 \mathrm{a}$ & $3.33 \mathrm{~d}$ & $4.00 \mathrm{~b}$ & $3.00 \mathrm{c}$ & $3.33 \mathrm{~b}$ \\
\hline & $1 \%$ & $5.00 \mathrm{a}$ & $5.00 \mathrm{a}$ & $5.00 \mathrm{a}$ & $5.00 \mathrm{a}$ & $4.00 \mathrm{c}$ & $5.00 \mathrm{a}$ & $3.33 \mathrm{~d}$ & $4.00 \mathrm{~b}$ & $2.67 \mathrm{~b}$ & $3.33 \mathrm{~d}$ \\
\hline
\end{tabular}

"Gloss Score: $5=$ none, $4=$ slight, $3=$ moderate, $2=$ severe, $1=$ extreme.

Egypt. J. Hort. Vol. 42, No.1 (2015) 
Data presented in Table 4 show the influence of hot water, chitosan and storage temperature on shriveling of sweet pepper during storage period. Sweet peppers appeared normal without any symptoms of shriveling among different treatments within 7 and 14 days of storage at $5^{\circ} \mathrm{C}$ or $8^{\circ} \mathrm{C}$. However, after 28 days of storage there were significant differences between all treatments and untreated control in shriveling (symptoms). Sweet peppers dipped in hot water at $45^{\circ} \mathrm{C}$ and sprayed with chitosan at the two concentrations and stored at $5^{\circ} \mathrm{C}$ or $8^{\circ} \mathrm{C}$ provided the lowest value of shriveling, while untreated control stored at 5 or $8^{\circ} \mathrm{C}$ had the highest values. These results are in agreement with those obtained by Maalekuu et al. (2005), who found that sweet peppers treated with hot water at $55^{\circ} \mathrm{C}$ for $15 \mathrm{sec}$. significantly reduced shriveling and maintained their appearance during storage. Also, Rodriguez et al. (2005) reported that the overall visual quality significantly improved by hot water treatment at both 2 and $8^{\circ} \mathrm{C}$ storage conditions for the 28 day storage. For chitosan treatment, Raymond et al. (2012) indicated that chitosan treatment reduced respiration rate and improved external appearance of green pepper during storage at $5^{\circ} \mathrm{C}$. Also, Fard et al. (2010) found that treating green peppers with chitosan delayed the change in external quality, reduced water loss during storage and maintained quality of the fruit. Regardless of the treatments results revealed that the lower the temperature of storage, the less the percentage of shriveled fruits is achieved. Sweet peppers stored at $5^{\circ} \mathrm{C}$ were much better in reducing shriveling. Freshness of fruits and vegetables can be preserved by reducing respiration rate and transpiration in order to avoid rapid quality deterioration, and that is commonly achieved by reducing storage temperature. (Kader, 2002 and Hardenburg et al., 1990).

The results in Table 5 indicate that fruit texture decreased progressively as the storage period was prolonged. Similar results are reported by Nora (2013) and Abdalla (2013) on sweet peppers. Data also showed that significant differences were found between hot water and chitosan treatments on fruit texture after 28 days of storage. However, sweet peppers dipped in hot water at $45^{\circ} \mathrm{C}$ and chitosan at 0.5 or $1 \%$ and stored at $5^{\circ} \mathrm{C}$ or $8^{\circ} \mathrm{C}$ had significantly greater fruit texture with no significant differences between fruit stored at $5^{\circ} \mathrm{C}$ or $8^{\circ} \mathrm{C}$ as compared with the other treatments and the untreated control. Hot water at $55^{\circ} \mathrm{C}$ + chitosan at the two concentrations and storage at 5 or $8^{\circ} \mathrm{C}$ were less effective in reducing the loss of texture. Comparing the effect of hot water and chitosan in maintaining hardness, of mango fruit stored at low temperature, Djioua et al. (2010) found that hot water dips at $50^{\circ} \mathrm{C}$ for $30 \mathrm{~min}$. was more effective than chitosan coating $(0.25 \%)$ during storage at $6^{\circ} \mathrm{C}$. However, hot water and chitosan treatment reduced the hardness loss during storage compared with chitosan alone or control. The mechanism by which pre-storage heating may affect the cell structure and thus maintain fruit firmness was explained by Vicente et al. (2003) who found that heat treated strawberries $\left(45^{\circ} \mathrm{C}\right.$ for $3 \mathrm{~h}$.) showed delay in softening by reduction of endo-1,4-B-D glueanse, B-xylosidasepolyglactonase and B-glactosidase activities, while pectin methyl esterase activity was enhanced. This heat treatments effect on cell wall degrading enzymes could slow down pectin solubilisation by reducing pectin cleavage and by increasing the amount of putative sites for calcium bridge formation in the cell wall. The

Egypt. J. Hort. Vol. 42, No.1 (2015) 
relatively greater texture of sweet peppers dipped in hot water is probably due to recrystallization or "melting" of the wax layer which scaled barely visible cracks (Fallik et al., 2005). Xing et al. (2011) found that chitosan treatment of peppers had significantly lower malondialdehyde (MDA) contents and relative leakage rates, as an indicator of membrane integrity, than the control fruit, indicating that higher membrane integrity was maintained.

TABLE 4. Effect of hot water, chitosan treatments and storage temperature on shriveling (score)* of sweet red pepper fruits (Testy red $\mathbf{F}_{1}$ Hybrid) during cold storage in 2011/2012 and 2012/2013 seasons.

\begin{tabular}{|c|c|c|c|c|c|c|c|c|c|c|c|}
\hline \multirow{2}{*}{\multicolumn{2}{|c|}{ Treatments }} & \multicolumn{10}{|c|}{ Storage period (days) } \\
\hline & & \multicolumn{2}{|c|}{ 0 } & \multicolumn{2}{|c|}{7} & \multicolumn{2}{|c|}{14} & \multicolumn{2}{|c|}{21} & \multicolumn{2}{|c|}{28} \\
\hline & & \multicolumn{10}{|c|}{2011 / 2012} \\
\hline 离 & 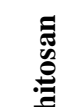 & $5^{\circ} \mathrm{C}$ & $8^{\circ} \mathbf{C}$ & $5^{\circ} \mathrm{C}$ & $8^{\circ} \mathrm{C}$ & $5^{\circ} \mathrm{C}$ & $8^{\circ} \mathrm{C}$ & $5^{\circ} \mathrm{C}$ & $8^{\circ} \mathrm{C}$ & $5^{\circ} \mathrm{C}$ & $8^{\circ} \mathrm{C}$ \\
\hline \multirow{3}{*}{$\begin{array}{c}\text { Control } \\
25^{\circ} \mathrm{c}\end{array}$} & 0 & $1.00 \mathrm{a}$ & $1.00 \mathrm{a}$ & $1.00 \mathrm{a}$ & $1.00 \mathrm{a}$ & $1.00 \mathrm{a}$ & $1.00 \mathrm{a}$ & $2.00 \mathrm{~d}$ & $2.66 \mathrm{a}$ & $3.00 \mathrm{~b}$ & $3.66 \mathrm{a}$ \\
\hline & $0.5 \%$ & $1.00 \mathrm{a}$ & $1.00 \mathrm{a}$ & $1.00 \mathrm{a}$ & $1.00 \mathrm{a}$ & $1.00 \mathrm{a}$ & $1.00 \mathrm{a}$ & $1.66 \mathrm{e}$ & $2.00 \mathrm{c}$ & $2.66 \mathrm{c}$ & $2.66 \mathrm{c}$ \\
\hline & $1 \%$ & $1.00 \mathrm{a}$ & $1.00 \mathrm{a}$ & $1.00 \mathrm{a}$ & $1.00 \mathrm{a}$ & $1.00 \mathrm{a}$ & $1.00 \mathrm{a}$ & $1.66 \mathrm{e}$ & $2.00 \mathrm{~d}$ & $2.66 \mathrm{c}$ & $2.66 \mathrm{c}$ \\
\hline \multirow{3}{*}{$45^{\circ} \mathrm{C}$} & 0 & $1.00 \mathrm{a}$ & $1.00 \mathrm{a}$ & $1.00 \mathrm{a}$ & $1.00 \mathrm{a}$ & $1.00 \mathrm{a}$ & $1.00 \mathrm{a}$ & $1.66 \mathrm{e}$ & $2.00 \mathrm{~d}$ & $2.33 \mathrm{~d}$ & $3.00 \mathrm{~b}$ \\
\hline & $0.5 \%$ & $1.00 \mathrm{a}$ & $1.00 \mathrm{a}$ & $1.00 \mathrm{a}$ & $1.00 \mathrm{a}$ & $1.00 \mathrm{a}$ & $1.00 \mathrm{a}$ & $1.00 \mathrm{~g}$ & $1.33 \mathrm{f}$ & $1.66 \mathrm{f}$ & $1.66 \mathrm{f}$ \\
\hline & $1 \%$ & $1.00 \mathrm{a}$ & $1.00 \mathrm{a}$ & $1.00 \mathrm{a}$ & $1.00 \mathrm{a}$ & $1.00 \mathrm{a}$ & $1.00 \mathrm{a}$ & $1.00 \mathrm{~g}$ & $1.66 \mathrm{e}$ & $1.66 \mathrm{f}$ & $1.66 \mathrm{f}$ \\
\hline \multirow{3}{*}{$55^{\circ} \mathrm{C}$} & 0 & $1.00 \mathrm{a}$ & $1.00 \mathrm{a}$ & $1.00 \mathrm{a}$ & $1.00 \mathrm{a}$ & $1.00 \mathrm{a}$ & $1.00 \mathrm{a}$ & $2.00 \mathrm{~d}$ & $2.33 \mathrm{~b}$ & $2.33 \mathrm{~d}$ & $2.66 \mathrm{c}$ \\
\hline & $0.5 \%$ & $1.00 \mathrm{a}$ & $1.00 \mathrm{a}$ & $1.00 \mathrm{a}$ & $1.00 \mathrm{a}$ & $1.00 \mathrm{a}$ & $1.00 \mathrm{a}$ & $1.33 \mathrm{f}$ & $1.66 \mathrm{e}$ & $2.60 \mathrm{c}$ & $2.33 \mathrm{e}$ \\
\hline & $1 \%$ & $1.00 \mathrm{a}$ & $1.00 \mathrm{a}$ & $1.00 \mathrm{a}$ & $1.00 \mathrm{a}$ & $1.00 \mathrm{a}$ & $1.00 \mathrm{a}$ & $1.33 \mathrm{f}$ & $1.66 \mathrm{e}$ & $2.00 \mathrm{e}$ & $2.33 \mathrm{e}$ \\
\hline \multicolumn{12}{|c|}{$2012 / 2013$} \\
\hline \multirow{3}{*}{$\begin{array}{c}\text { Control } \\
25^{\circ} \mathrm{c}\end{array}$} & 0 & $1.00 \mathrm{a}$ & $1.00 \mathrm{a}$ & $1.00 \mathrm{a}$ & $1.00 \mathrm{a}$ & $1.00 \mathrm{a}$ & $1.00 \mathrm{a}$ & $2.33 \mathrm{~b}$ & $2.66 \mathrm{a}$ & $3.33 \mathrm{~b}$ & $3.66 \mathrm{a}$ \\
\hline & $0.5 \%$ & $1.00 \mathrm{a}$ & $1.00 \mathrm{a}$ & $1.00 \mathrm{a}$ & $1.00 \mathrm{a}$ & $1.00 \mathrm{a}$ & $1.00 \mathrm{a}$ & $2.00 \mathrm{c}$ & $2.33 \mathrm{~b}$ & $2.33 \mathrm{e}$ & $3.00 \mathrm{c}$ \\
\hline & $1 \%$ & $1.00 \mathrm{a}$ & $1.00 \mathrm{a}$ & $1.00 \mathrm{a}$ & $1.00 \mathrm{a}$ & $1.00 \mathrm{a}$ & $1.00 \mathrm{a}$ & $1.66 \mathrm{~d}$ & $2.00 \mathrm{c}$ & $2.66 \mathrm{~d}$ & $3.00 \mathrm{c}$ \\
\hline \multirow{3}{*}{$45^{\circ} \mathrm{C}$} & 0 & $1.00 \mathrm{a}$ & $1.00 \mathrm{a}$ & $1.00 \mathrm{a}$ & $1.00 \mathrm{a}$ & $1.00 \mathrm{a}$ & $1.00 \mathrm{a}$ & $2.00 \mathrm{~d}$ & $2.33 \mathrm{~b}$ & $2.66 \mathrm{~d}$ & $3.00 \mathrm{c}$ \\
\hline & $0.5 \%$ & $1.00 \mathrm{a}$ & $1.00 \mathrm{a}$ & $1.00 \mathrm{a}$ & $1.00 \mathrm{a}$ & $1.00 \mathrm{a}$ & $1.00 \mathrm{a}$ & $1.00 \mathrm{f}$ & $1.33 \mathrm{e}$ & $1.66 \mathrm{~g}$ & $2.00 \mathrm{f}$ \\
\hline & $1 \%$ & $1.00 \mathrm{a}$ & $1.00 \mathrm{a}$ & $1.00 \mathrm{a}$ & $1.00 \mathrm{a}$ & $1.00 \mathrm{a}$ & $1.00 \mathrm{a}$ & $1.00 \mathrm{f}$ & $1.66 \mathrm{~d}$ & $1.66 \mathrm{~g}$ & $2.33 \mathrm{e}$ \\
\hline \multirow{3}{*}{$55^{\circ} \mathrm{C}$} & 0 & $1.00 \mathrm{a}$ & $1.00 \mathrm{a}$ & $1.00 \mathrm{a}$ & $1.00 \mathrm{a}$ & $1.00 \mathrm{a}$ & $1.00 \mathrm{a}$ & $2.00 \mathrm{c}$ & $2.66 \mathrm{a}$ & $2.66 \mathrm{~d}$ & $3.00 \mathrm{c}$ \\
\hline & $0.5 \%$ & $1.00 \mathrm{a}$ & $1.00 \mathrm{a}$ & $1.00 \mathrm{a}$ & $1.00 \mathrm{a}$ & $1.00 \mathrm{a}$ & $1.00 \mathrm{a}$ & $1.33 \mathrm{e}$ & $1.66 \mathrm{~d}$ & $2.00 \mathrm{f}$ & 2.66 \\
\hline & $1 \%$ & $1.00 \mathrm{a}$ & $1.00 \mathrm{a}$ & $1.00 \mathrm{a}$ & $1.00 \mathrm{a}$ & $1.00 \mathrm{a}$ & $1.00 \mathrm{a}$ & $1.33 \mathrm{e}$ & $2.00 \mathrm{c}$ & $2.00 \mathrm{f}$ & $2.66 \mathrm{~d}$ \\
\hline
\end{tabular}

Shriveling Score: $1=$ none, $2=$ slight, $3=$ moderate, $4=$ severe, $5=$ extreme. 
TABLE 5. Effect of hot water, chitosan treatments and storage temperature on fruit texture $\left(\mathrm{p} / \mathrm{En}^{2}\right)$ of sweet pepper fruits (Testy red $\mathrm{F}_{1}$ Hybrid) during cold storage in 2011/2012 and 2012/2013 seasons.

\begin{tabular}{|c|c|c|c|c|c|c|c|c|c|c|c|}
\hline \multirow{3}{*}{\multicolumn{2}{|c|}{ Treatments }} & \multicolumn{10}{|c|}{ Storage period (days) } \\
\hline & & \multicolumn{2}{|c|}{$\overline{0}$} & \multicolumn{2}{|c|}{7} & \multicolumn{2}{|c|}{14} & \multicolumn{2}{|c|}{21} & \multicolumn{2}{|c|}{28} \\
\hline & & \multicolumn{10}{|c|}{$2011 / 2012$} \\
\hline 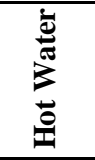 & 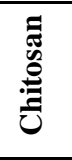 & $5^{\circ} \mathrm{C}$ & $8^{\circ} \mathrm{C}$ & $5^{\circ} \mathbf{C}$ & $\mathbf{8}^{\circ} \mathbf{C}$ & $5^{\circ} \mathrm{C}$ & $8^{\circ} \mathrm{C}$ & $\mathbf{5}^{\circ} \mathbf{C}$ & $8^{\circ} \mathbf{C}$ & $5^{\circ} \mathrm{C}$ & $8^{\circ} \mathrm{C}$ \\
\hline \multirow{3}{*}{$\begin{array}{c}\text { Control } \\
25^{\circ} \mathrm{c}\end{array}$} & 0 & $3.33 \mathrm{a}$ & $3.33 \mathrm{a}$ & $3.00 \mathrm{bc}$ & $2.90 \mathrm{c}$ & $2.81 \mathrm{bf}$ & $2.60 \mathrm{f}$ & $2.40 \mathrm{j}$ & $2.53 \mathrm{ch}$ & $1.80 \mathrm{j}$ & $2.24 \mathrm{ch}$ \\
\hline & $0.5 \%$ & $3.33 \mathrm{a}$ & $3.33 \mathrm{a}$ & $3.14 \mathrm{ab}$ & $3.10 \mathrm{ac}$ & $2.87 \mathrm{ad}$ & $2.74 \mathrm{cf}$ & $2.48 \mathrm{hi}$ & $2.65 \mathrm{ef}$ & $2.00 \mathrm{I}$ & $2.35 \mathrm{ef}$ \\
\hline & $1 \%$ & $3.33 \mathrm{a}$ & $3.33 \mathrm{a}$ & $3.13 \mathrm{ac}$ & $3.12 \mathrm{ac}$ & $2.70 \mathrm{df}$ & $2.62 \mathrm{ef}$ & $2.45 \mathrm{ij}$ & $2.61 \mathrm{ef}$ & $2.00 \mathrm{I}$ & $2.31 \mathrm{e}-\mathrm{g}$ \\
\hline \multirow{3}{*}{$45^{\circ} \mathrm{C}$} & 0 & $3.33 \mathrm{a}$ & $3.33 \mathrm{a}$ & $3.16 \mathrm{ab}$ & $3.10 \mathrm{ac}$ & $2.94 \mathrm{ac}$ & $2.82 \mathrm{bf}$ & $2.50 \mathrm{hi}$ & $2.74 \mathrm{~cd}$ & $2.32 \mathrm{ef}$ & $2.54 \mathrm{~b}$ \\
\hline & $0.5 \%$ & $3.33 \mathrm{a}$ & $3.33 \mathrm{a}$ & $3.29 \mathrm{a}$ & $3.19 \mathrm{ab}$ & $3.10 \mathrm{a}$ & $3.00 \mathrm{ab}$ & $2.81 \mathrm{bc}$ & $2.91 \mathrm{a}$ & $2.59 \mathrm{ab}$ & $2.65 \mathrm{a}$ \\
\hline & $1 \%$ & $3.33 \mathrm{a}$ & $3.33 \mathrm{a}$ & $3.25 \mathrm{a}$ & $3.11 \mathrm{ac}$ & $3.00 \mathrm{ab}$ & $2.94 \mathrm{ac}$ & $2.65 \mathrm{ef}$ & $2.82 \mathrm{~b}$ & $2.55 \mathrm{~b}$ & $2.60 \mathrm{ab}$ \\
\hline \multirow{3}{*}{$55^{\circ} \mathrm{C}$} & 0 & $3.33 \mathrm{a}$ & $3.33 \mathrm{a}$ & $3.08 \mathrm{acc}$ & $2.98 \mathrm{bc}$ & $2.90 \mathrm{ad}$ & $2.70 \mathrm{df}$ & $2.53 \mathrm{gh}$ & $2.60 \mathrm{fg}$ & $2.20 \mathrm{~h}$ & $2.35 \mathrm{ef}$ \\
\hline & $0.5 \%$ & $3.33 \mathrm{a}$ & $3.33 \mathrm{a}$ & $3.20 \mathrm{ab}$ & $3.17 \mathrm{ab}$ & $2.90 \mathrm{ad}$ & 2.85 be & $2.68 \mathrm{de}$ & $2.76 \mathrm{bc}$ & $2.38 \mathrm{de}$ & $2.46 \mathrm{c}$ \\
\hline & $1 \%$ & $3.33 \mathrm{a}$ & $3.33 \mathrm{a}$ & $3.18 \mathrm{ab}$ & $3.10 \mathrm{ac}$ & $2.80 \mathrm{bff}$ & $2.80 \mathrm{b-f}$ & $2.64 \mathrm{ef}$ & $2.68 \mathrm{de}$ & $2.30 \mathrm{fg}$ & $2.44 \mathrm{~cd}$ \\
\hline \multicolumn{12}{|c|}{$2012 / 2013$} \\
\hline \multirow{3}{*}{$\begin{array}{c}\text { Control } \\
25^{\circ} \mathrm{c}\end{array}$} & 0 & $4.41 \mathrm{a}$ & $4.41 \mathrm{a}$ & $4.06 \mathrm{~cd}$ & $3.83 \mathrm{f}$ & $3.85 \mathrm{cf}$ & $3.62 \mathrm{f}$ & $3.20 \mathrm{f}$ & $3.30 \mathrm{ef}$ & $2.50 \mathrm{~h}$ & $3.00 \mathrm{ef}$ \\
\hline & $0.5 \%$ & $4.41 \mathrm{a}$ & $4.41 \mathrm{a}$ & $4.10 \mathrm{c}$ & $3.92 \mathrm{e}$ & $3.94 \mathrm{ad}$ & $3.71 \mathrm{df}$ & $3.40 \mathrm{cff}$ & $3.48 \mathrm{ag}$ & $2.83 \mathrm{gg}$ & $3.18 \mathrm{ce}$ \\
\hline & $1 \%$ & $4.41 \mathrm{a}$ & $4.41 \mathrm{a}$ & $4.11 \mathrm{bc}$ & $3.87 \mathrm{ef}$ & $3.90 \mathrm{be}$ & $3.70 \mathrm{ef}$ & $3.38 \mathrm{cff}$ & 3.45 be & $2.76 \mathrm{~g}$ & $3.14 \mathrm{ce}$ \\
\hline \multirow{3}{*}{$45^{\circ} \mathrm{C}$} & 0 & $4.41 \mathrm{a}$ & $4.41 \mathrm{a}$ & $4.18 \mathrm{~b}$ & $4.00 \mathrm{~d}$ & $4.00 \mathrm{ac}$ & $3.80 \mathrm{cf}$ & $3.49 \mathrm{re}$ & $3.55 \mathrm{ad}$ & $2.86 \mathrm{gg}$ & 3.21 be \\
\hline & $0.5 \%$ & $4.41 \mathrm{a}$ & $4.41 \mathrm{a}$ & $4.26 \mathrm{a}$ & $4.11 \mathrm{bc}$ & $4.15 \mathrm{a}$ & $3.92 \mathrm{at}$ & $3.66 \mathrm{ab}$ & $3.70 \mathrm{a}$ & $3.36 \mathrm{ac}$ & $3.50 \mathrm{a}$ \\
\hline & $1 \%$ & $4.41 \mathrm{a}$ & $4.41 \mathrm{a}$ & $4.30 \mathrm{a}$ & $4.12 \mathrm{bc}$ & $4.10 \mathrm{ab}$ & $3.80 \mathrm{cf}$ & $3.67 \mathrm{ab}$ & $3.68 \mathrm{ab}$ & $3.35 \mathrm{ac}$ & $3.43 \mathrm{ab}$ \\
\hline \multirow{3}{*}{$55^{\circ} \mathrm{C}$} & 0 & $4.41 \mathrm{a}$ & $4.41 \mathrm{a}$ & $4.10 \mathrm{c}$ & $3.91 \mathrm{e}$ & $3.90 \mathrm{be}$ & $3.72 \mathrm{df}$ & $3.35 \mathrm{df}$ & $3.45 \mathrm{be}$ & $2.72 \mathrm{ch}$ & $3.10 \mathrm{de}$ \\
\hline & $0.5 \%$ & $4.41 \mathrm{a}$ & $4.41 \mathrm{a}$ & $4.18 \mathrm{~b}$ & $4.10 \mathrm{c}$ & $4.00 \mathrm{acc}$ & $3.80 \mathrm{cf}$ & $3.56 \mathrm{ad}$ & $3.60 \mathrm{ac}$ & $3.18 \mathrm{ce}$ & $3.30 \mathrm{ad}$ \\
\hline & $1 \%$ & $4.41 \mathrm{a}$ & $4.41 \mathrm{a}$ & $4.09 \mathrm{c}$ & $4.00 \mathrm{~d}$ & $4.00 \mathrm{acc}$ & $3.81 \mathrm{cf}$ & $3.41 \mathrm{cff}$ & $3.52 \mathrm{ae}$ & $3.00 \mathrm{ef}$ & $3.20 \mathrm{be}$ \\
\hline
\end{tabular}

Concerning the influence of hot water, chitosan and storage temperature on pitting of sweet peppers during storage period, data in Table 6 indicate that fruits stored at $8^{\circ} \mathrm{C}$ did not show any pitting during all storage period. This disorder occurred only in sweet peppers stored at $5^{\circ} \mathrm{C}$ after 14 days of storage and became pronounced after 28 days of storage. However, sweet pepper fruits treated with hot water at $45^{\circ} \mathrm{C}+$ chitosan at the two concentrations and kept at $5^{\circ} \mathrm{C}$ did not develop any pitting after 21 days of storage and gave non to traces of pitting on the surface after 28 days of storage. On the other hand, sweet peppers treated with hot water at $55^{\circ} \mathrm{C}+$ two concentrations of chitos an and stored at $5^{\circ} \mathrm{C}$ resulted in traces of pitting at the end of storage period ( 28 days), while untreated control had severe incidence during the same period. These results are in accordance with those obtained by Lim et al. (2007) as they found that sweet peppers stored below $7^{\circ} \mathrm{C}$ had CI within several days. The optimum storage temperature recommended is between $8-10{ }^{\circ} \mathrm{C}$ for sweet pepper (Kader, 1986, Kehr (2002). Abdul Hakim (1997) indicated that hot

Egypt. J. Hort. Vol. 42, No.1 (2015) 
water at $46^{\circ} \mathrm{C}$ for $90 \mathrm{~min}$ could allow longer storage of mature green tomato by lowering CI, $\mathrm{CO}_{2}$, ethylene production, and electrolyte leakage. Lurie et al. (1986) reported a correlation between the development of heat shock protein during storage and prevention of CI, expressed as pitting on the surface of the fruit. Sapitnitskaya et al. (2006).suggested that postharvest heat treatments induced fruit tolerance to cold temperature and reduced the development of chilling injury CI symptoms during cold storage.

TABLE 6. Effect of hot water, chitosan treatments and storage temperature on pitting(score)* of sweet pepper fruits (Testy red $F_{1}$ Hybrid) during cold storage in 2011/2012 and 2012/2013 seasons.

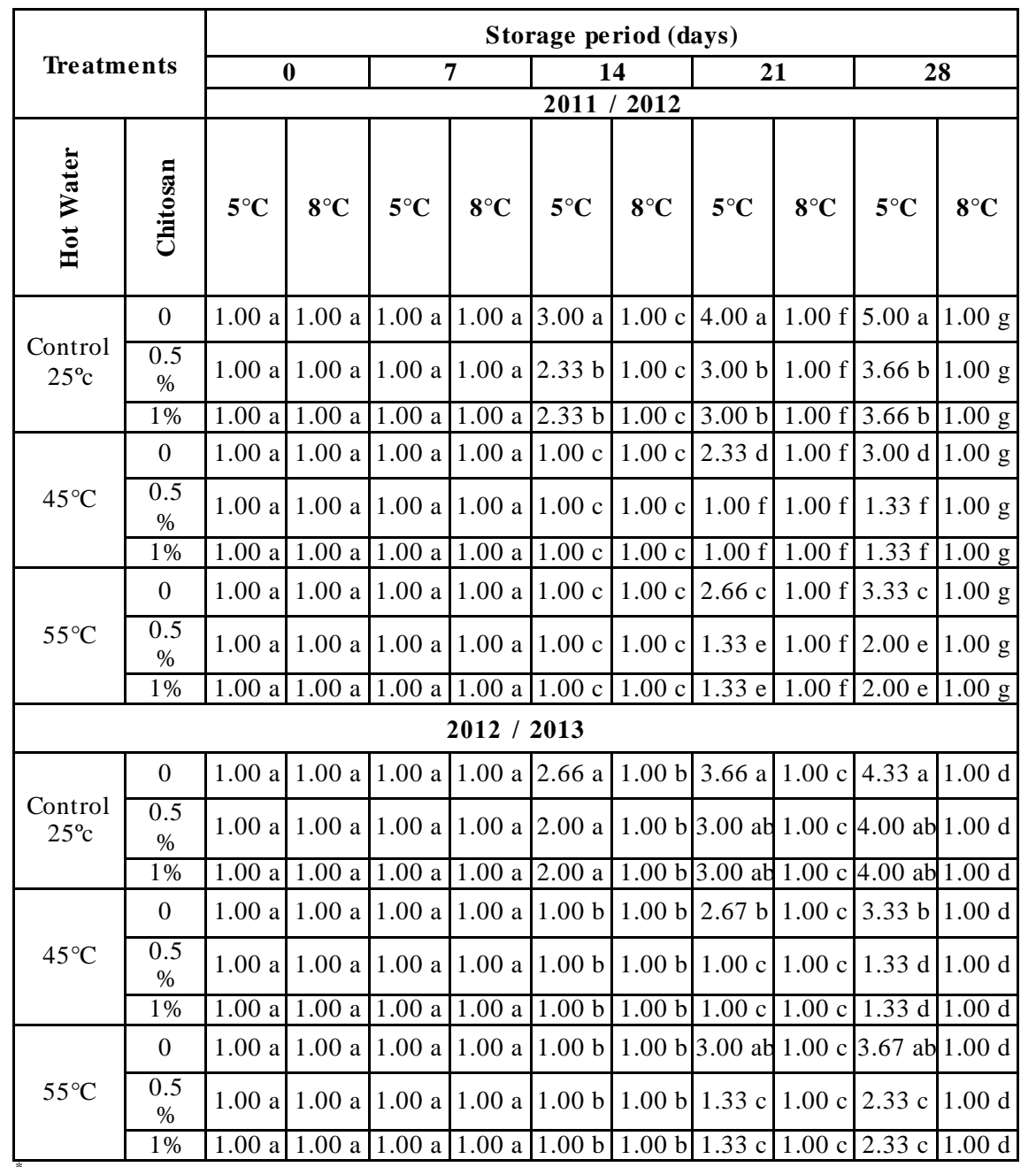

"Pitting Score: 1 = no abnormality, $2=$ trace, $3=$ slight, $4=$ moderate and $5=$ severe. 
Table 7 present the influence of hot water, chitosan and storage temperature on calyx darkening. No calyx darkening were observed in all treatments and control after 7 and 14 days of storage at $5^{\circ} \mathrm{C}$ and $8^{\circ} \mathrm{C}$ and after 21 days at $8^{\circ} \mathrm{C}$. Sweet peppers treated with hot water at $45^{\circ} \mathrm{C}+$ chitos an at 0.5 and $1 \%$ and kept at $5^{\circ} \mathrm{C}$ did not develop any calyx darkening after 21 days of storage and gave non to traces of this character after 28 days of storage. Sweet peppers which dipped in hot water at $45^{\circ} \mathrm{C}+$ two chitosan concentrations and stored at $8^{\circ} \mathrm{C}$ appeared normal without any symptoms of calyx darkening after 28 days of storage. These results are in agreement with those of Shehata et al. (2009), which revealed that sweet peppers dipped in hot water had none to slight discoloration after 9 days of storage at $4^{\circ} \mathrm{C}$. Kehr (2002) reported that hot water treatments at $60^{\circ} \mathrm{C}$ for $1 \mathrm{~min}$. reduced the development of $\mathrm{CI}$ and maintained the appearance of sweet peppers during cold storage. The favorable effect of hot water treatment in reducing CI may be due to that heat treatment induces the production of heat shock proteins (HSP) which are involved in chilling prevention (Sabehat et al., 1996, Porat et al., 2000 and Zhang et al., 2005).High relative amount of HSP in peppers enabled a better defense mechanism against CI (Bar-Yosef et al., 2009). Concerning the effect of chitosan treatment, MengMeng et al. (2012) reported that treating summer squash fruit with chitosan coating could postpone the appearance of CI symptoms.

Results of seed darkening are given in Table (8) peppers stored at $8^{\circ} \mathrm{C}$ did not show any seed darkening during all storage periods. Concerning the peppers stored at $5^{\circ} \mathrm{C}$, significant differences in seed darkening between all treatments and control was detected after 21 and 28 days of storage. However, peppers treated with hot water at $45^{\circ} \mathrm{C}+$ chitosan at the two concentrations and stored at $5^{\circ} \mathrm{C}$ did not show any seed darkening till 21 days of storage and had non to traces of this character after 28 days of storage. These results are in agreement with those of, Abd-EL-Rahman (1990) who found that sweet peppers stored at $10^{\circ} \mathrm{C}$ did not develop any symptoms of CI till the end of storage period (30 days).According to Kader (1986) the optimum storage temperature of sweet peppers range between $8-10^{\circ} \mathrm{C}$. Pre-storage heat treatments have been reported to induce fruit tolerance to cold temperature and to reduce the development of CI symptoms of sweet pepper fruits during cold storage (Bar-Yosef et al., 2009 and Mencareli et al., 1993).Hot water treatments of $53^{\circ} \mathrm{C}$ for $3 \mathrm{~min}$. for mandarin fruit reduced catalase activity during cold storage which was associated with increased CI (Sala and Lafuentc, 2000) suggested that hot water treatment $\left(53^{\circ} \mathrm{C}\right)$ for $3 \mathrm{~min}$. reduced catalase activity which was associated with the increase in CI symptoms during cold storage of mandarin. Concerning the effect of chitosan, MengMeng et al. (2012) found that chitosan reduced the occurrence of summer squash CI during storage.

Egypt. J. Hort. Vol. 42, No.1 (2015) 
TABLE 7. Effect of hot water, chitosan treatments and storage temperature on calyx darkening (score) * of sweet pepper fruits (Testy red $\mathbf{F}_{1}$ Hybrid) during cold storage in 2011/2012 and 2012/ 2013 seasons.

\begin{tabular}{|c|c|c|c|c|c|c|c|c|c|c|c|}
\hline \multirow{3}{*}{\multicolumn{2}{|c|}{ Treatments }} & \multicolumn{10}{|c|}{ Storage period (days) } \\
\hline & & \multicolumn{2}{|c|}{$\mathbf{0}$} & \multicolumn{2}{|c|}{7} & \multicolumn{2}{|c|}{14} & \multicolumn{2}{|c|}{21} & \multicolumn{2}{|c|}{28} \\
\hline & & \multicolumn{10}{|c|}{$2011 / 2012$} \\
\hline 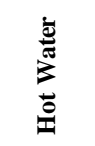 & 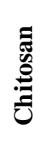 & $5^{\circ} \mathrm{C}$ & $8^{\circ} \mathrm{C}$ & $5^{\circ} \mathrm{C}$ & $8^{\circ} \mathrm{C}$ & $5^{\circ} \mathrm{C}$ & $8^{\circ} \mathrm{C}$ & $5^{\circ} \mathrm{C}$ & $8^{\circ} \mathrm{C}$ & $5^{\circ} \mathrm{C}$ & $8^{\circ} \mathrm{C}$ \\
\hline \multirow{3}{*}{$\begin{array}{c}\text { Control } \\
25^{\circ} \mathrm{c}\end{array}$} & 0 & $1.00 \mathrm{a}$ & $1.00 \mathrm{a}$ & $1.00 \mathrm{a}$ & $1.00 \mathrm{a}$ & $1.00 \mathrm{a}$ & $1.00 \mathrm{a}$ & $3.00 \mathrm{a}$ & $1.00 \mathrm{c}$ & $4.33 \mathrm{a}$ & $2.33 \mathrm{ce}$ \\
\hline & $\begin{array}{c}0.5 \\
\%\end{array}$ & $1.00 \mathrm{a}$ & $1.00 \mathrm{a}$ & $1.00 \mathrm{a}$ & $1.00 \mathrm{a}$ & $1.00 \mathrm{a}$ & $1.00 \mathrm{a}$ & $2.33 \mathrm{ab}$ & $1.00 \mathrm{c}$ & $3.33 \mathrm{~b}$ & $1.83 \mathrm{ef}$ \\
\hline & $1 \%$ & $1.00 \mathrm{a}$ & $1.00 \mathrm{a}$ & $1.00 \mathrm{a}$ & $1.00 \mathrm{a}$ & $1.00 \mathrm{a}$ & $1.00 \mathrm{a}$ & $2.33 \mathrm{ab}$ & $1.00 \mathrm{c}$ & $3.00 \mathrm{bc}$ & 1.83 ef \\
\hline \multirow{3}{*}{$45^{\circ} \mathrm{C}$} & 0 & $1.00 \mathrm{a}$ & $1.00 \mathrm{a}$ & $1.00 \mathrm{a}$ & $1.00 \mathrm{a}$ & $1.00 \mathrm{a}$ & $1.00 \mathrm{a}$ & $2.33 \mathrm{ab}$ & $1.00 \mathrm{c}$ & $2.66 \mathrm{~b}-\mathrm{d}$ & d 1.66 ef \\
\hline & $\begin{array}{c}0.5 \\
\%\end{array}$ & $1.00 \mathrm{a}$ & $1.00 \mathrm{a}$ & $1.00 \mathrm{a}$ & $1.00 \mathrm{a}$ & $1.00 \mathrm{a}$ & $1.00 \mathrm{a}$ & $1.00 \mathrm{c}$ & $1.00 \mathrm{c}$ & $1.66 \mathrm{ef}$ & $1.00 \mathrm{f}$ \\
\hline & $1 \%$ & $1.00 \mathrm{a}$ & $1.00 \mathrm{a}$ & $1.00 \mathrm{a}$ & $1.00 \mathrm{a}$ & $1.00 \mathrm{a}$ & $1.00 \mathrm{a}$ & $1.00 \mathrm{c}$ & $1.00 \mathrm{c}$ & $1.66 \mathrm{ef}$ & $1.00 \mathrm{f}$ \\
\hline \multirow{3}{*}{$55^{\circ} \mathrm{C}$} & 0 & $1.00 \mathrm{a}$ & $1.00 \mathrm{a}$ & $1.00 \mathrm{a}$ & $1.00 \mathrm{a}$ & $1.00 \mathrm{a}$ & $1.00 \mathrm{a}$ & $2.33 \mathrm{ab}$ & $1.00 \mathrm{c}$ & $3.00 \mathrm{bc}$ & $2.00 \mathrm{~d}-\mathrm{f}$ \\
\hline & $\begin{array}{c}0.5 \\
\%\end{array}$ & $1.00 \mathrm{a}$ & $1.00 \mathrm{a}$ & $1.00 \mathrm{a}$ & $1.00 \mathrm{a}$ & $1.00 \mathrm{a}$ & $1.00 \mathrm{a}$ & $2.00 \mathrm{~b}$ & $1.00 \mathrm{c}$ & $2.66 \mathrm{~b}-\mathrm{d}$ & d 1.66 ef \\
\hline & $1 \%$ & $1.00 \mathrm{a}$ & $1.00 \mathrm{a}$ & $1.00 \mathrm{a}$ & $1.00 \mathrm{a}$ & $1.00 \mathrm{a}$ & $1.00 \mathrm{a}$ & $2.00 \mathrm{~b}$ & $1.00 \mathrm{c}$ & $2.33 \mathrm{c}-\mathrm{e}$ & 1.66 ef \\
\hline \multicolumn{12}{|c|}{$2012 / 2013$} \\
\hline \multirow{3}{*}{$\begin{array}{c}\text { Control } \\
25^{\circ} \mathrm{c}\end{array}$} & 0 & $1.00 \mathrm{a}$ & $1.00 \mathrm{a}$ & $1.00 \mathrm{a}$ & $1.00 \mathrm{a}$ & $1.00 \mathrm{a}$ & $1.00 \mathrm{a}$ & $2.66 \mathrm{a}$ & $1.00 \mathrm{c}$ & $4.00 \mathrm{a}$ & $2.33 \mathrm{~cd}$ \\
\hline & $\begin{array}{c}0.5 \\
\% \\
\end{array}$ & $1.00 \mathrm{a}$ & $1.00 \mathrm{a}$ & $1.00 \mathrm{a}$ & $1.00 \mathrm{a}$ & $1.00 \mathrm{a}$ & $1.00 \mathrm{a}$ & $2.00 \mathrm{ab}$ & $1.00 \mathrm{c}$ & $3.33 \mathrm{ab}$ & $1.66 \mathrm{~d}-\mathrm{f}$ \\
\hline & $1 \%$ & $1.00 \mathrm{a}$ & $1.00 \mathrm{a}$ & $1.00 \mathrm{a}$ & $1.00 \mathrm{a}$ & $1.00 \mathrm{a}$ & $1.00 \mathrm{a}$ & $2.00 \mathrm{ab}$ & $1.00 \mathrm{c}$ & $3.00 \mathrm{bc}$ & $1.66 \mathrm{~d}-\mathrm{f}$ \\
\hline \multirow{3}{*}{$45^{\circ} \mathrm{C}$} & 0 & $1.00 \mathrm{a}$ & $1.00 \mathrm{a}$ & $1.00 \mathrm{a}$ & $1.00 \mathrm{a}$ & $1.00 \mathrm{a}$ & $1.00 \mathrm{a}$ & $2.00 \mathrm{ab}$ & $1.00 \mathrm{c}$ & $3.00 \mathrm{bc}$ & $1.66 \mathrm{~d}-\mathrm{f}$ \\
\hline & $\begin{array}{c}0.5 \\
\%\end{array}$ & $1.00 \mathrm{a}$ & $1.00 \mathrm{a}$ & $1.00 \mathrm{a}$ & $1.00 \mathrm{a}$ & $1.00 \mathrm{a}$ & $1.00 \mathrm{a}$ & $1.00 \mathrm{c}$ & $1.00 \mathrm{c}$ & 1.33 ef & $1.00 \mathrm{f}$ \\
\hline & $1 \%$ & $1.00 \mathrm{a}$ & $1.00 \mathrm{a}$ & $1.00 \mathrm{a}$ & $1.00 \mathrm{a}$ & $1.00 \mathrm{a}$ & $1.00 \mathrm{a}$ & $1.00 \mathrm{c}$ & $1.00 \mathrm{c}$ & $1.33 \mathrm{ef}$ & $1.00 \mathrm{f}$ \\
\hline \multirow{3}{*}{$55^{\circ} \mathrm{C}$} & 0 & $1.00 \mathrm{a}$ & $1.00 \mathrm{a}$ & $1.00 \mathrm{a}$ & $1.00 \mathrm{a}$ & $1.00 \mathrm{a}$ & $1.00 \mathrm{a}$ & $2.33 \mathrm{ab}$ & $1.00 \mathrm{c}$ & $3.33 \mathrm{ab}$ & $2.00 \mathrm{de}$ \\
\hline & $\begin{array}{c}0.5 \\
\%\end{array}$ & $1.00 \mathrm{a}$ & $1.00 \mathrm{a}$ & $1.00 \mathrm{a}$ & $1.00 \mathrm{a}$ & $1.00 \mathrm{a}$ & $1.00 \mathrm{a}$ & $1.66 \mathrm{bc}$ & $1.00 \mathrm{c}$ & $2.00 \mathrm{de}$ & $1.33 \mathrm{de}$ \\
\hline & $1 \%$ & $1.00 \mathrm{a}$ & $1.00 \mathrm{a}$ & $1.00 \mathrm{a}$ & $1.00 \mathrm{a}$ & $1.00 \mathrm{a}$ & $1.00 \mathrm{a}$ & $1.66 \mathrm{bc}$ & $1.00 \mathrm{c}$ & $2.33 \mathrm{ef}$ & $1.33 \mathrm{~cd}$ \\
\hline
\end{tabular}

"Calyx darkening Score:1 = no abnormality, $2=$ trace, $3=$ slight, $4=$ moderate and $5=$ severe. 
TABLE 8. Effect of hot water, chitosan treatments and storage temperature on seed darkening (score) ${ }^{*}$ of sweet pepper fruits (Testy red $F_{1}$ Hybrid) during cold storage in 2011/2012 and 2012/2013 seasons.

\begin{tabular}{|c|c|c|c|c|c|c|c|c|c|c|c|}
\hline \multirow{3}{*}{\multicolumn{2}{|c|}{ Treatments }} & \multicolumn{10}{|c|}{ Storage period (days) } \\
\hline & & \multicolumn{2}{|c|}{$\mathbf{0}$} & \multicolumn{2}{|c|}{7} & \multicolumn{2}{|c|}{14} & \multicolumn{2}{|c|}{21} & \multicolumn{2}{|c|}{28} \\
\hline & & \multicolumn{10}{|c|}{$2011 / 2012$} \\
\hline$\stackrel{\vec{\theta}}{\frac{\grave{\Delta}}{\pi}}$ & 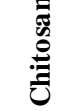 & $5^{\circ} \mathrm{C}$ & $8^{\circ} \mathrm{C}$ & $5^{\circ} \mathbf{C}$ & $8^{\circ} \mathrm{C}$ & $5^{\circ} \mathrm{C}$ & $8^{\circ} \mathrm{C}$ & $5^{\circ} \mathrm{C}$ & $8^{\circ} \mathrm{C}$ & $5^{\circ} \mathrm{C}$ & $8^{\circ} \mathbf{C}$ \\
\hline \multirow{3}{*}{$\begin{array}{c}\text { Control } \\
25^{\circ} \mathrm{c}\end{array}$} & 0 & $1.00 \mathrm{a}$ & $1.00 \mathrm{a}$ & $1.00 \mathrm{a}$ & $1.00 \mathrm{a}$ & $1.00 \mathrm{a}$ & $1.00 \mathrm{a}$ & $3.33 \mathrm{a}$ & $1.00 \mathrm{~g}$ & $4.66 \mathrm{a}$ & $1.00 \mathrm{~h}$ \\
\hline & $0.5 \%$ & $1.00 \mathrm{a}$ & $1.00 \mathrm{a}$ & $1.00 \mathrm{a}$ & $1.00 \mathrm{a}$ & $1.00 \mathrm{a}$ & $1.00 \mathrm{a}$ & $2.33 \mathrm{c}$ & $1.00 \mathrm{~g}$ & $3.33 \mathrm{c}$ & $1.00 \mathrm{~h}$ \\
\hline & $1 \%$ & $1.00 \mathrm{a}$ & $1.00 \mathrm{a}$ & $1.00 \mathrm{a}$ & $1.00 \mathrm{a}$ & $1.00 \mathrm{a}$ & $1.00 \mathrm{a}$ & $2.00 \mathrm{~d}$ & $1.00 \mathrm{~g}$ & $3.33 \mathrm{c}$ & $1.00 \mathrm{~h}$ \\
\hline \multirow{3}{*}{$45^{\circ} \mathrm{C}$} & 0 & $1.00 \mathrm{a}$ & $1.00 \mathrm{a}$ & $1.00 \mathrm{a}$ & $1.00 \mathrm{a}$ & $1.00 \mathrm{a}$ & $1.00 \mathrm{a}$ & $2.33 \mathrm{c}$ & $1.00 \mathrm{~g}$ & $3.66 \mathrm{~b}$ & $1.00 \mathrm{~h}$ \\
\hline & $0.5 \%$ & $1.00 \mathrm{a}$ & $1.00 \mathrm{a}$ & $1.00 \mathrm{a}$ & $1.00 \mathrm{a}$ & $1.00 \mathrm{a}$ & $1.00 \mathrm{a}$ & $1.00 \mathrm{~g}$ & $1.00 \mathrm{~g}$ & $1.33 \mathrm{~g}$ & $1.00 \mathrm{~h}$ \\
\hline & $1 \%$ & $1.00 \mathrm{a}$ & $1.00 \mathrm{a}$ & $1.00 \mathrm{a}$ & $1.00 \mathrm{a}$ & $1.00 \mathrm{a}$ & $1.00 \mathrm{a}$ & $1.00 \mathrm{~g}$ & $1.00 \mathrm{~g}$ & $1.66 \mathrm{f}$ & $1.00 \mathrm{~h}$ \\
\hline \multirow{3}{*}{$55^{\circ} \mathrm{C}$} & 0 & $1.00 \mathrm{a}$ & $1.00 \mathrm{a}$ & $1.00 \mathrm{a}$ & $1.00 \mathrm{a}$ & $1.00 \mathrm{a}$ & $1.00 \mathrm{a}$ & $2.66 \mathrm{~b}$ & $1.00 \mathrm{~g}$ & $3.66 \mathrm{~b}$ & $1.00 \mathrm{~h}$ \\
\hline & $0.5 \%$ & $1.00 \mathrm{a}$ & $1.00 \mathrm{a}$ & $1.00 \mathrm{a}$ & $1.00 \mathrm{a}$ & $1.00 \mathrm{a}$ & $1.00 \mathrm{a}$ & $1.33 \mathrm{f}$ & $1.00 \mathrm{~g}$ & $2.00 \mathrm{e}$ & $1.00 \mathrm{~h}$ \\
\hline & $1 \%$ & $1.00 \mathrm{a}$ & $1.00 \mathrm{a}$ & $1.00 \mathrm{a}$ & $1.00 \mathrm{a}$ & $1.00 \mathrm{a}$ & $1.00 \mathrm{a}$ & $1.66 \mathrm{e}$ & $1.00 \mathrm{~g}$ & $2.33 \mathrm{~d}$ & $1.00 \mathrm{~h}$ \\
\hline \multicolumn{12}{|c|}{$2012 / 2013$} \\
\hline \multirow{3}{*}{$\begin{array}{c}\text { Control } \\
25^{\circ} \mathrm{c}\end{array}$} & 0 & $1.00 \mathrm{a}$ & $1.00 \mathrm{a}$ & $1.00 \mathrm{a}$ & $1.00 \mathrm{a}$ & $1.00 \mathrm{a}$ & $1.00 \mathrm{a}$ & $3.00 \mathrm{a}$ & $1.00 \mathrm{e}$ & $4.33 \mathrm{a}$ & $1.00 \mathrm{~g}$ \\
\hline & $0.5 \%$ & $1.00 \mathrm{a}$ & $1.00 \mathrm{a}$ & $1.00 \mathrm{a}$ & $1.00 \mathrm{a}$ & $1.00 \mathrm{a}$ & $1.00 \mathrm{a}$ & $2.00 \mathrm{c}$ & $1.00 \mathrm{e}$ & $3.00 \mathrm{~d}$ & $1.00 \mathrm{~g}$ \\
\hline & $1 \%$ & $1.00 \mathrm{a}$ & $1.00 \mathrm{a}$ & $1.00 \mathrm{a}$ & $1.00 \mathrm{a}$ & $1.00 \mathrm{a}$ & $1.00 \mathrm{a}$ & $2.00 \mathrm{c}$ & $1.00 \mathrm{e}$ & $3.33 \mathrm{c}$ & $1.00 \mathrm{~g}$ \\
\hline \multirow{3}{*}{$45^{\circ} \mathrm{C}$} & 0 & $1.00 \mathrm{a}$ & $1.00 \mathrm{a}$ & $1.00 \mathrm{a}$ & $1.00 \mathrm{a}$ & $1.00 \mathrm{a}$ & $1.00 \mathrm{a}$ & $2.00 \mathrm{c}$ & $1.00 \mathrm{e}$ & $3.66 \mathrm{~b}$ & $1.00 \mathrm{~g}$ \\
\hline & $0.5 \%$ & $1.00 \mathrm{a}$ & $1.00 \mathrm{a}$ & $1.00 \mathrm{a}$ & $1.00 \mathrm{a}$ & $1.00 \mathrm{a}$ & $1.00 \mathrm{a}$ & $1.00 \mathrm{e}$ & $1.00 \mathrm{e}$ & $1.33 \mathrm{f}$ & $1.00 \mathrm{~g}$ \\
\hline & $1 \%$ & $1.00 \mathrm{a}$ & $1.00 \mathrm{a}$ & $1.00 \mathrm{a}$ & $1.00 \mathrm{a}$ & $1.00 \mathrm{a}$ & $1.00 \mathrm{a}$ & $1.00 \mathrm{e}$ & $1.00 \mathrm{e}$ & $1.33 \mathrm{f}$ & 1.00 \\
\hline \multirow{3}{*}{$55^{\circ} \mathrm{C}$} & 0 & $1.00 \mathrm{a}$ & $1.00 \mathrm{a}$ & $1.00 \mathrm{a}$ & $1.00 \mathrm{a}$ & $1.00 \mathrm{a}$ & $1.00 \mathrm{a}$ & $2.33 \mathrm{~b}$ & $1.00 \mathrm{e}$ & $3.66 \mathrm{~b}$ & $1.00 \mathrm{~g}$ \\
\hline & $0.5 \%$ & $1.00 \mathrm{a}$ & $1.00 \mathrm{a}$ & $1.00 \mathrm{a}$ & $1.00 \mathrm{a}$ & $1.00 \mathrm{a}$ & $1.00 \mathrm{a}$ & $1.66 \mathrm{~d}$ & $1.00 \mathrm{e}$ & $2.00 \mathrm{e}$ & $1.00 \mathrm{~g}$ \\
\hline & $1 \%$ & $1.00 \mathrm{a}$ & $1.00 \mathrm{a}$ & $1.00 \mathrm{a}$ & $1.00 \mathrm{a}$ & $1.00 \mathrm{a}$ & $1.00 \mathrm{a}$ & $1.66 \mathrm{~d}$ & $1.00 \mathrm{e}$ & $2.00 \mathrm{e}$ & 1.00 \\
\hline
\end{tabular}

Data presented in Table 9 show the influence of hot water, chitosan and storage temperature on carotenoids of sweet peppers during storage. There was no significant difference in total carotenoids contents among treatments including control after 7 days of storage. However, after 28 days of storage, the differences were significant. Sweet peppers treated with hot water at $45^{\circ} \mathrm{C}+$ chitosan at the two concentrations $(0.5$ and $1.0 \%$ ) and stored at $5^{\circ} \mathrm{C}$ had the highest carotenoids content compared with untreated control stored at 5 or $8^{\circ} \mathrm{C}$. Peppers treated with hot water at $45^{\circ} \mathrm{C}+$ chitosan at 0.5 and $1.0 \%$ and stored at $5^{\circ} \mathrm{C}$ had 2.94 and 2.90 $\mathrm{mg} / 100 \mathrm{~g}$ F.W with no significant differences between them. In general sweet peppers stored at $5^{\circ} \mathrm{C}$ had the highest total carotenoids content, 
while those stored at $8^{\circ} \mathrm{C}$ had the lowest content. These results agree with those of Fallik (2004) and Shehata et al. (2004) for hot water and Fard et al. (2010) and Xing et al. (2011) for chitosan. These results agree with Abd-EL-Rahman (1990) who found that sweet peppers stored at 1 and $4^{\circ} \mathrm{C}$ reduced the loss of color and delayed the degradation of green color during storage. Lowering the temperature of non-climacteric fruits like sweet peppers lowers their rate of ripening and deterioration (Kays, 1991) and maintains the retention of green color observed on peppers stored at 4 and $6.5^{\circ} \mathrm{C}$ (Nyanjage et al., 2005).

TABLE 9. Effect of hot water, chitosan treatments and storage temperature on carotenoids content $(\mathrm{mg} / \mathbf{1 0 0 g}$ fresh weight)of sweet pepper fruits (Testy red $F_{1}$ Hybrid)during cold storage in 2011/2012 and 2012/2013 seasons.

\begin{tabular}{|c|c|c|c|c|c|c|c|c|c|c|c|}
\hline \multirow{3}{*}{\multicolumn{2}{|c|}{ Treatments }} & \multicolumn{10}{|c|}{ Storage period (days) } \\
\hline & & \multicolumn{2}{|c|}{ 0 } & \multicolumn{2}{|c|}{7} & \multicolumn{2}{|c|}{14} & \multicolumn{2}{|c|}{21} & \multicolumn{2}{|c|}{28} \\
\hline & & \multicolumn{10}{|c|}{$2011 / 2012$} \\
\hline 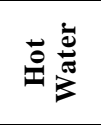 & 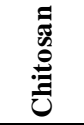 & $5^{\circ} \mathrm{C}$ & $8^{\circ} \mathrm{C}$ & $5^{\circ} \mathrm{C}$ & $8^{\circ} \mathrm{C}$ & $5^{\circ} \mathrm{C}$ & $8^{\circ} \mathrm{C}$ & $5^{\circ} \mathrm{C}$ & $8^{\circ} \mathrm{C}$ & $5^{\circ} \mathrm{C}$ & $8^{\circ} \mathrm{C}$ \\
\hline \multirow{3}{*}{$\begin{array}{c}\text { Control } \\
25^{\circ} \mathrm{c}\end{array}$} & 0 & $2.50 \mathrm{a}$ & $2.50 \mathrm{a}$ & $2.62 \mathrm{ab}$ & $2.66 \mathrm{ab}$ & $2.75 \mathrm{c}-\mathrm{f}$ & $2.87 \mathrm{a}$ & $2.80 \mathrm{e}-\mathrm{g}$ & $\mathrm{g} 2.70 \mathrm{~h}$ & $2.30 \mathrm{~h}$ & $2.22 \mathrm{I}$ \\
\hline & $0.5 \%$ & $2.50 \mathrm{a}$ & $2.50 \mathrm{a}$ & $2.60 \mathrm{ab}$ & $2.63 \mathrm{ab}$ & $2.70 \mathrm{f}$ & $2.80 \mathrm{a}-\mathrm{d}$ & $2.73 \mathrm{gh}$ & $\mathrm{h} 2.80 \mathrm{f}-\mathrm{l}$ & $2.50 \mathrm{~cd}$ & $\mathrm{~d} 2.62 \mathrm{~cd}$ \\
\hline & $1 \%$ & $2.50 \mathrm{a}$ & $2.50 \mathrm{a}$ & $2.61 \mathrm{ab}$ & $2.63 \mathrm{ab}$ & $2.73 \mathrm{df}$ & $2.82 \mathrm{a}-\mathrm{c}$ & $2.70 \mathrm{~h}$ & $2.86 \mathrm{de}$ & $2.46 \mathrm{fg}$ & $2.40 \mathrm{~g}$ \\
\hline \multirow{3}{*}{$45^{\circ} \mathrm{C}$} & 0 & $2.50 \mathrm{a}$ & $2.50 \mathrm{a}$ & $2.61 \mathrm{ab}$ & $2.64 \mathrm{ab}$ & $2.75 \mathrm{c}-\mathrm{f}$ & $2.85 \mathrm{ab}$ & $2.97 \mathrm{~b}$ & $3.00 \mathrm{~b}$ & $2.60 \mathrm{~cd}$ & $2.50 \mathrm{ef}$ \\
\hline & $0.5 \%$ & $2.50 \mathrm{a}$ & $2.50 \mathrm{a}$ & $2.59 \mathrm{~b}$ & $2.63 \mathrm{ab}$ & $2.69 \mathrm{f}$ & $2.80 \mathrm{a}-\mathrm{d}$ & $2.86 \mathrm{de}$ & $2.94 \mathrm{bc}$ & $2.94 \mathrm{a}$ & $2.73 \mathrm{~b}$ \\
\hline & $1 \%$ & $2.50 \mathrm{a}$ & $2.50 \mathrm{a}$ & $2.60 \mathrm{ab}$ & $2.64 a b$ & $2.74 \mathrm{dff}$ & $2.83 a b$ & $2.87 \mathrm{c}-\mathrm{C}$ & $3.16 \mathrm{a}$ & $2.90 \mathrm{a}$ & $2.62 \mathrm{~cd}$ \\
\hline \multirow{3}{*}{$55^{\circ} \mathrm{C}$} & 0 & $2.50 \mathrm{a}$ & $2.50 \mathrm{a}$ & $2.63 \mathrm{ab}$ & $2.66 \mathrm{ab}$ & $2.75 \mathrm{c}-\mathrm{f}$ & $2.83 \mathrm{ab}$ & $2.93 \mathrm{~b}-\mathrm{c}$ & $\mathrm{d} 2.99 \mathrm{~b}$ & $2.55 \mathrm{de}$ & $2.48 \mathrm{ef}$ \\
\hline & $0.5 \%$ & $2.50 \mathrm{a}$ & $2.50 \mathrm{a}$ & $2.65 \mathrm{ab}$ & $2.67 \mathrm{a}$ & $2.72 \mathrm{ef}$ & $2.79 \mathrm{be}$ & $2.84 \mathrm{ef}$ & $2.95 \mathrm{~b}$ & $2.79 \mathrm{~b}$ & $2.65 \mathrm{c}$ \\
\hline & $1 \%$ & $2.50 \mathrm{a}$ & $2.50 \mathrm{a}$ & $2.62 \mathrm{ab}$ & $2.65 a b$ & $2.70 \mathrm{f}$ & $2.80 \mathrm{a}-\mathrm{d}$ & $2.85 \mathrm{e}$ & $2.94 \mathrm{bc}$ & $2.78 \mathrm{~b}$ & $2.60 \mathrm{~cd}$ \\
\hline \multicolumn{12}{|c|}{$2012 / 2013$} \\
\hline \multirow{3}{*}{$\begin{array}{c}\text { Control } \\
25^{\circ} \mathrm{c}\end{array}$} & 0 & $2.70 \mathrm{a}$ & $2.70 \mathrm{a}$ & $2.72 \mathrm{fg}$ & $2.72 \mathrm{fg}$ & $3.32 \mathrm{~g}$ & $3.52 \mathrm{~cd}$ & $3.33 \mathrm{j}$ & $3.53 \mathrm{gh}$ & $3.18 \mathrm{k}$ & $3.30 \mathrm{j}$ \\
\hline & $0.5 \%$ & $2.70 \mathrm{a}$ & $2.70 \mathrm{a}$ & $2.78 \mathrm{~b}-1$ & $2.77 \mathrm{c}-\mathrm{g}$ & $3.48 \mathrm{de}$ & $3.71 \mathrm{a}$ & $3.48 \mathrm{hi}$ & $3.74 \mathrm{c}$ & $3.40 \mathrm{hi}$ & \begin{tabular}{ll|}
$\mathrm{i}$ & $3.33 \mathrm{ij}$ \\
\end{tabular} \\
\hline & $1 \%$ & $2.70 \mathrm{a}$ & $2.70 \mathrm{a}$ & $2.75 \mathrm{e}-$ & $2.76 \mathrm{dg}$ & $3.56 \mathrm{bc}$ & $3.46 \mathrm{df}$ & $3.60 \mathrm{eg}$ & $3.53 \mathrm{gh}$ & $3.40 \mathrm{hi}$ & \begin{tabular}{|l|l|}
$\mathrm{i}$ & $3.33 \mathrm{ij}$ \\
\end{tabular} \\
\hline \multirow{3}{*}{$45^{\circ} \mathrm{C}$} & 0 & $2.70 \mathrm{a}$ & $2.70 \mathrm{a}$ & $2.77 \mathrm{c}-$ & $2.73 \mathrm{e}-\mathrm{g}$ & $3.43 \mathrm{ef}$ & $3.40 \mathrm{f}$ & $3.49 \mathrm{bc}$ & $3.44 \mathrm{i}$ & $3.50 \mathrm{fg}$ & $3.36 \mathrm{ij}$ \\
\hline & $0.5 \%$ & $2.70 \mathrm{a}$ & $2.70 \mathrm{a}$ & $2.84 \mathrm{a}-\mathrm{c}$ & $2.84 \mathrm{a}-\mathrm{c}$ & $3.50 \mathrm{c}-\mathrm{e}$ & $3.43 \mathrm{ef}$ & $3.86 \mathrm{ab}$ & $3.89 \mathrm{a}$ & $3.90 \mathrm{a}$ & $3.60 \mathrm{de}$ \\
\hline & $1 \%$ & $2.70 \mathrm{a}$ & $2.70 \mathrm{a}$ & $2.84 \mathrm{a}-\mathrm{d}$ & $2.83 \mathrm{a}-\mathrm{d}$ & $3.50 \mathrm{c}-\mathrm{e}$ & $3.46 c-f$ & $3.82 \mathrm{ab}$ & $3.82 \mathrm{ab}$ & $3.83 \mathrm{a}$ & $3.64 \mathrm{~cd}$ \\
\hline \multirow{3}{*}{$55^{\circ} \mathrm{C}$} & 0 & $2.70 \mathrm{a}$ & $2.70 \mathrm{a}$ & $2.71 \mathrm{fg}$ & $2.70 \mathrm{~g}$ & $3.53 \mathrm{~cd}$ & $3.50 \mathrm{c}-\mathrm{e}$ & $3.64 \mathrm{ef}$ & $3.58 \mathrm{eg}$ & $3.45 \mathrm{gh}$ & $\mathrm{h} 3.36 \mathrm{ij}$ \\
\hline & $0.5 \%$ & $2.70 \mathrm{a}$ & $2.70 \mathrm{a}$ & $1.92 \mathrm{~h}$ & $2.85 \mathrm{ab}$ & $3.63 \mathrm{~b}$ & $3.56 \mathrm{bc}$ & $3.72 \mathrm{~cd}$ & $3.65 \mathrm{de}$ & $3.70 \mathrm{bc}$ & $3.52 \mathrm{fg}$ \\
\hline & $1 \%$ & $2.70 \mathrm{a}$ & $2.70 \mathrm{a}$ & $2.87 \mathrm{a}$ & $2.80 \mathrm{a}-\mathrm{e}$ & $3.40 \mathrm{f}$ & $3.53 \mathrm{~cd}$ & $3.45 \mathrm{i}$ & $3.57 \mathrm{fg}$ & $3.72 \mathrm{~b}$ & $3.55 \mathrm{ef}$ \\
\hline
\end{tabular}


Significant differences were detected between hot water, chitosan treatments and different temperatures in total soluble solids content after 28 days of storage (Table10). However, sweet peppers dipped in hot water at $45^{\circ} \mathrm{C}$ and chitosan at $0.5 \%$ stored at $5^{\circ} \mathrm{C}$ exhibited highest TSS content compared with untreated control which stored at $5^{\circ} \mathrm{C}$ or $8^{\circ} \mathrm{C}$. These results agree with those of Sakaldas and Kaynas (2010) who found that sweet peppers treated with hot water had higher TSS contents than the control.The higher levels of TSS in the sweet peppers coated with chitosan may be due to the reduction of the oxygen supply on the fruit surface, which reduced respiration, thus slowing the changes of TSS contents (Yonemato et al., 2002).

TABLE 10. Effect of hot water, chitosan treatments and storage temperature on total soluble solids \% of sweet pepper fruits (Testy red $F_{1}$ Hybrid)during cold storage in 2011/2012 and 2012/2013 seasons.

\begin{tabular}{|c|c|c|c|c|c|c|c|c|c|c|c|}
\hline \multirow{3}{*}{\multicolumn{2}{|c|}{ Treatments }} & \multicolumn{10}{|c|}{ Storage period (days) } \\
\hline & & \multicolumn{2}{|c|}{$\mathbf{0}$} & \multicolumn{2}{|c|}{7} & \multicolumn{2}{|c|}{14} & \multicolumn{2}{|c|}{21} & \multicolumn{2}{|c|}{28} \\
\hline & & \multicolumn{10}{|c|}{$2011 / 2012$} \\
\hline $\overrightarrow{0}$ & 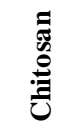 & $5^{\circ} \mathrm{C}$ & $8^{\circ} \mathrm{C}$ & $5^{\circ} \mathrm{C}$ & $8^{\circ} \mathrm{C}$ & $5^{\circ} \mathrm{C}$ & $8^{\circ} \mathrm{C}$ & $5^{\circ} \mathrm{C}$ & $8^{\circ} \mathrm{C}$ & $5^{\circ} \mathrm{C}$ & $8^{\circ} \mathrm{C}$ \\
\hline \multirow{3}{*}{$\begin{array}{c}\text { Contr } \\
\text { ol } \\
25^{\circ} \mathrm{C}\end{array}$} & 0 & $9.06 \mathrm{a}$ & $9.06 \mathrm{a}$ & $8.83 \mathrm{a}-\mathrm{c}$ & $8.72 \mathrm{c}$ & $8.62 \mathrm{bc}$ & $8.41 \mathrm{~d}$ & $8.33 \mathrm{gh}$ & $8.11 \mathrm{j}$ & $7.83 \mathrm{i}$ & $7.52 \mathrm{k}$ \\
\hline & $0.5 \%$ & $9.06 \mathrm{a}$ & $9.06 \mathrm{a}$ & $8.90 \mathrm{ab}$ & $8.84 a-c$ & $8.74 \mathrm{ab}$ & $8.60 \mathrm{bc}$ & $8.52 \mathrm{~cd}$ & $8.38 \mathrm{~h}$ & $8.16 \mathrm{~b}-\mathrm{c}$ & $8.02 \mathrm{fg}$ \\
\hline & $1 \%$ & $9.06 \mathrm{a}$ & $9.06 \mathrm{a}$ & $8.92 \mathrm{ab}$ & $8.80 \mathrm{a}-\mathrm{c}$ & $8.70 \mathrm{ab}$ & $8.70 \mathrm{ab}$ & $8.57 \mathrm{bc}$ & $8.42 \mathrm{f}$ & $8.10 \mathrm{de}$ & $7.95 \mathrm{~g}$ \\
\hline \multirow{3}{*}{$45^{\circ} \mathrm{C}$} & 0 & $9.06 \mathrm{a}$ & $9.06 \mathrm{a}$ & $8.91 \mathrm{ab}$ & $8.82 \mathrm{a}-\mathrm{c}$ & $8.75 \mathrm{ab}$ & $8.52 \mathrm{cc}$ & $8.53 \mathrm{c}$ & $8.31 \mathrm{gh}$ & $8.02 \mathrm{fg}$ & $8.11 \mathrm{cc}$ \\
\hline & $0.5 \%$ & $9.06 \mathrm{a}$ & $9.06 \mathrm{a}$ & $8.95 \mathrm{a}$ & $8.91 \mathrm{ab}$ & $8.82 \mathrm{a}$ & $8.72 \mathrm{ab}$ & $8.64 \mathrm{ab}$ & $8.43 \mathrm{ef}$ & $\begin{array}{ll}\mathrm{f} & 8.35 \mathrm{a}\end{array}$ & $8.18 \mathrm{bc}$ \\
\hline & $1 \%$ & $9.06 \mathrm{a}$ & $9.06 \mathrm{a}$ & $8.90 \mathrm{ab}$ & $8.88 \mathrm{a}-\mathrm{c}$ & $8.74 \mathrm{ab}$ & $8.62 \mathrm{bc}$ & $8.51 \mathrm{~cd}$ & $8.32 \mathrm{gh}$ & $8.20 \mathrm{~b}$ & $8.03 \mathrm{ef}$ \\
\hline \multirow{4}{*}{$55^{\circ} \mathrm{C}$} & 0 & $9.06 \mathrm{a}$ & $9.06 \mathrm{a}$ & $8.87 \mathrm{a}-\mathrm{c}$ & $8.80 \mathrm{a}-\mathrm{c}$ & $8.70 \mathrm{ab}$ & $8.53 \mathrm{cc}$ & $8.50 \mathrm{c}-\mathrm{c}$ & $8.20 \mathrm{i}$ & $7.93 \mathrm{~h}$ & $7.65 \mathrm{j}$ \\
\hline & $0.5 \%$ & $9.06 \mathrm{a}$ & $9.06 \mathrm{a}$ & $8.92 \mathrm{ab}$ & $8.87 \mathrm{a}-\mathrm{c}$ & $8.85 \mathrm{a}$ & $8.72 \mathrm{ab}$ & $8.65 \mathrm{a}$ & $8.45 \mathrm{~d}-$ & $8.20 \mathrm{~b}$ & $8.03 \mathrm{ef}$ \\
\hline & $1 \%$ & $9.06 \mathrm{a}$ & $9.06 \mathrm{a}$ & $8.90 \mathrm{ab}$ & $8.77 b c$ & $8.70 \mathrm{ab}$ & $8.60 \mathrm{bc}$ & $8.52 \mathrm{~cd}$ & $8.38 \mathrm{fg}$ & $8.10 \mathrm{df}$ & $7.92 \mathrm{~h}$ \\
\hline & & \multicolumn{10}{|c|}{$2012 / 2013$} \\
\hline \multirow{3}{*}{$\begin{array}{c}\text { Contr } \\
\text { ol } \\
25^{\circ} \mathrm{c}\end{array}$} & 0 & $8.50 \mathrm{a}$ & $8.50 \mathrm{a}$ & 8.2 & $8.11 \mathrm{a}-\mathrm{c}$ & $8.10 \mathrm{~b}-\mathrm{c}$ & $8.00 \mathrm{~d}$ & $7.61 \mathrm{c}$ & $7.62 \mathrm{c}$ & $7.25 \mathrm{~d}-\mathrm{g}$ & $6.80 \mathrm{j}$ \\
\hline & $0.5 \%$ & $8.50 \mathrm{a}$ & $8.50 \mathrm{a}$ & $8.40 \mathrm{ab}$ & $8.20 \mathrm{a}-\mathrm{c}$ & $8.28 \mathrm{a}-\mathrm{c}$ & $8.11 \mathrm{~b}-\mathrm{d}$ & $7.90 \mathrm{c}$ & $7.52 \mathrm{c}$ & $7.50 \mathrm{bc}$ & $7.12 \mathrm{f}-\mathrm{i}$ \\
\hline & $1 \%$ & $8.50 \mathrm{a}$ & $8.50 \mathrm{a}$ & $8.41 \mathrm{ab}$ & $8.16 \mathrm{a}-\mathrm{c}$ & $8.20 \mathrm{a}-\mathrm{c}$ & $8.10 \mathrm{~b}-\mathrm{d}$ & $7.84 \mathrm{c}$ & $7.41 \mathrm{c}$ & $7.45 b-d$ & $6.90 \mathrm{ij}$ \\
\hline \multirow{3}{*}{$45^{\circ} \mathrm{C}$} & 0 & $8.50 \mathrm{a}$ & $8.50 \mathrm{a}$ & $8.35 \mathrm{ab}$ & $8.19 \mathrm{a}-\mathrm{c}$ & $8.30 \mathrm{a}-\mathrm{c}$ & $8.20 \mathrm{a}-\mathrm{d}$ & $8.78 \mathrm{a}$ & $7.84 \mathrm{c}$ & $7.40 \mathrm{c}-\mathrm{e}$ & e $7.20 \mathrm{e}-\mathrm{h}$ \\
\hline & $0.5 \%$ & $8.50 \mathrm{a}$ & $8.50 \mathrm{a}$ & $8.42 \mathrm{a}$ & $8.30 \mathrm{ab}$ & $8.36 \mathrm{a}$ & $8.20 \mathrm{a}-\mathrm{d}$ & $8.10 \mathrm{a}-\mathrm{c}$ & $7.70 \mathrm{c}$ & $7.88 \mathrm{a}$ & $7.30 \mathrm{c}-1$ \\
\hline & $1 \%$ & $8.50 \mathrm{a}$ & $8.50 \mathrm{a}$ & $8.31 \mathrm{a}-\mathrm{c}$ & $8.20 \mathrm{a}-\mathrm{c}$ & $8.32 \mathrm{ab}$ & $8.14 a-d$ & $8.08 \mathrm{a}-\mathrm{c}$ & $7.52 \mathrm{c}$ & $7.80 \mathrm{a}$ & $7.20 \mathrm{e}-\mathrm{h}$ \\
\hline \multirow{3}{*}{$55^{\circ} \mathrm{C}$} & 0 & $8.50 \mathrm{a}$ & $8.50 \mathrm{a}$ & $8.27 \mathrm{a}-\mathrm{c}$ & $8.00 \mathrm{c}$ & $8.12 b-c$ & $8.10 b-d$ & $8.64 \mathrm{ab}$ & $7.72 \mathrm{c}$ & $7.31 \mathrm{c}-1$ & $\mathrm{f} 7.06 \mathrm{~g}-$ \\
\hline & $0.5 \%$ & $8.50 \mathrm{a}$ & $8.50 \mathrm{a}$ & $8.38 \mathrm{ab}$ & $8.17 \mathrm{a}-\mathrm{c}$ & $8.30 \mathrm{a}-\mathrm{c}$ & $8.07 \mathrm{~cd}$ & $8.00 \mathrm{bc}$ & $7.62 \mathrm{c}$ & $7.65 \mathrm{ab}$ & $7.14 \mathrm{f}-\mathrm{h}$ \\
\hline & $1 \%$ & $8.50 \mathrm{a}$ & $8.50 \mathrm{a}$ & $8.28 \mathrm{a}-\mathrm{c}$ & $8.10 \mathrm{bc}$ & $8.17 \mathrm{a}-\mathrm{c}$ & $8.00 \mathrm{~d}$ & $8.00 \mathrm{bc}$ & $7.41 \mathrm{c}$ & $7.52 \mathrm{bc}$ & c7.00h-j \\
\hline
\end{tabular}

Egypt. J. Hort. Vol. 42, No.1 (2015) 


\section{Conclusion}

Sweet peppers treated with hot water at $45^{\circ} \mathrm{C}+$ chitosan at 0.5 or $1 \%$ stored at $5^{\circ} \mathrm{C}$ had less $\mathrm{CI}$ and better quality during storage.

\section{References}

A.O.A.C. (1990) Quality of Official Analy tical Chemists, Washington D.C., USA.

Abd El-Rahman, S.Z. (1990) Physiological Studies on Development, Handling and Storage of Pepper. Ph.D. Thesis, Fac. Agric., Al-Azhar Univ., Egy pt, 154 p.

Abdalla, M.A.A. (2013) Pre and Postharvest Treatments to Enhance Sweet Pepper (Capsicum annuum L.)Productivity and Quality. Ph.D. Thesis, Fac. Agric., Ain Shams Univ., Cairo, Egypt, 101 p.

Abdul Hakim, A., Kaukovirate, E., Pehu, E. and Voipio., l. (1997) Effect of hot water, immersion time, and length of storage on chilling injury of tomato fruit. Journal of vegetable crop production, $3(2)$.

Bar-Yosef, A., Alkalai-Tuvia, S., Perzelan, Y., Aharon, Z., Ilic, Z., Lurie, S. and Fallik, E. (2009) Effect of shrink packaging in combination with rinsing and brushing treatment on chilling injury and decay of sweet pepper during storage. Advanced Horticulture Science, 23, 225 -230.

Bayoumi, Y.A. (2008) Improvement of postharvest keeping quality of white pepper fruits (Capsicum annum L.)by hydrogen peroxide treatment under storage conditions. Acta Biologica Szegediensis, 52 (1), 7-15.

Chiloo, M.J. and Asgharim, M.R. (2011) Hot water and chitosan treatment for the control of postharvest decay in sweet cherry (Prunusavium L.) CV. Napoleon (Napoly on). Journal of stored products and postharvest Research 2 (7), 135-138, July 2011.

Djioua, T., Charles, F., Freire, M., Filgueiras, H., Ducamp-Collin, M.N. and Sallanon, H. (2010) Combined effects of postharvest heat treatments and chitosan coating on quality of fresh-cut mangoes (Mangifera indica L.) International Journal of Food Science and Technology. 45, 849-855.

Dong, H., Cheng, L., Tan, and Jiang, Y. (2004) Effect of chitosan coating on quality and shelf-life of peeled litchi fruit. J. Food Eng., 64, 355-358.

EL-Badawy, M.S.A. (2014) Effect of Spray with some safety compounds on growth and productivity of some strawberry cultivars. M.SC.Thesis, Fac.Agric., Banha Univ.,Egy pt,76 p.

Fajardo, J.E., McCollum, T.G., McDonald, R.E. and Mayer, R.T., (1998) Differential induction of proteins induction of proteins in orange flaved by biologically based elicitors and challenged penicilliumdigitatumsacc. Biol.Control, 13,143-151. 
Fallik, E. (2004) Prestorage hot water treatments (immersion, rinsing and brushing). Postharvest Biology and Technology, 32, 125-134.

Fallik, E., Bar-Yosef, A. and Alkalai-Tuvia, Sh. (2009) Prevention of chilling injury in sweet bell pepper stored at $1.5 \mathrm{c}$ by heat treatments and individual shrink packaging. Folia Horticulturae, 21, 87-97.

Fallik, E., Shalom, Y., Alkalai-Tuvia, S., Larkov, O., Brandeis, E. and Ravid, U. (2005) External, internal and sensory trais in Gala-type melon tyrated with different waxes. Postharvest Bio. Technol., 36, 69-75.

Fallik, E., Grinberg, S. G., Alkalai, S., Yekutieli, O., Wiseblum, A., Regev, R., Beres, H. and Bar-Lev, E. (1999) A unique rapid hot water treatment to improve storage quality of sweet pepper. Postharvest Biology and Technology, 15, 25-32.

Fard, K.G., Kamari, M. Ghasemnezhad and Ghazvini, R.F. (2010) Effect of chitosan coating on weight loss and postharvest quality of Green pepper (Capsicum annumL.) Fruits. Acta Hort., 877, ISHS 2010.

Gonzalez-Aguilar, G., Cruz, R., Baez, R. and Wang, C.Y. (1999) Storage quality of bell peppers pretreated with hot water and polyethylene packaging. J. Food Qual., 22 (3), 287-299.

Hardenburg, R.E., Watada, A. and Wang, C.Y. (1990) The commercial storage of fruits, vegetables, florist and nursery stocks. Washington, DC: US Department of Agriculture, Agriculture Handbook, No. 66. pp. 23-25.

Howard, L.R., Talcott, S.T., Brenes, C.H. and Villalon, B. (2000) Changes in phytochochemical and antioxidant activity of selected pepper cultivars (Capsicum species) as influenced by maturity. Journal of Agricultural and Food Chemistry, 48, 1713-1720.

Ilic, Z.S., Radmila, T. Rados, P. Sharon, A.T. Yaacov, P. and Elazar, F. (2012) Effect of heat treatment and individual shrink packaging on quality and nutrition value ofbell pepper stored at suboptimal temperature. International Journal of Food Science and Technology, 47, 83-90.

Kader, A.A. (1986) Biochemical and physiological basis for effects of controlled and modified atmospheres on fruits and vegetables. Food Technology, 40(5), 99-104.

Kader, A.A. (2002) Postharvest technology of horticultural crops. University of California, Division of Agriculture and Natural Resources Publication, 3311, 535.

Kays, S. J. (1991) Secondary metabolic processes and products : An overview. In S.J. Kays (Ed.) Postharvest physiology of perishable plant products.Univ. Georgia Athens., pp. 187-213.

Kehr, M.E. (2002) Susceptibility to postharvest chilling damage in sweet peppers,and treatments to minimize its effect. Agricultura Tecnica, 62(4), 497-508.32 ref. 
Lim, C.S., Kang, S.M., Cho, J.L., Gross, K.C. and Woolf, A.B. (2007) Bell pepper (Capsicum annuumL.) fruits are susceptible to chilling injury at the breaker stage of ripening. HortScience, 42, 1659-1664.

Lurie, S., Shapiro, B., Ben-Yehoshua, S. (1986) Effects of water stress and degree of ripeness on rate of senescence of harvested bell pepper fruit. J. AM. Soc. Hort. Sci., 111, 880-885.

Maalekuu, K., ElkInd, Y., Tvvia-Alkalai, S., Shalom, Y. and Fallik, E. (2003) Quality evaluation of three sweet pepper cultivars after prolonged storage. Adv. Hort. Sci., 17 (4), 187-191.

Maalekuu K., Tuvia Alkalars, Shalomy, Y., El-kind, Y. and Fallik, E. (2005) Characterization of physiological and biochemical factors associated with postharvest water loss in rip pepper fruit during storage. J. Amer..Soc., 130, 745-741.

Mencareli, F., Ceccantoni, B., Bolini, A. and Anelli, G. (1993) Influence of heat treatment on the physiological response of sweet pepper kept at chilling temperature. Acta Hort., 343, 238-243.

MengMeng, Y., Lipu, G., Qing, W., Jie, P. and Na (2012) Effect of chitosan coating on chilling injury in summer squash. Jornalof Henan Agricultural Scineces, 41 (10), 114-117.12 ref.

NORA, A.G. El-Rab (2013) Effect of some pre and postharvest treatments on yield, quality and storability of Sweet Pepper Productivity and Quality. M.Sc. Thesis, Fac. Agric., Cairo Univ., Cairo, Egypt, 101 p.

Nyanjage, M.O., Nyalala, S.P.O., Illa, A.O., Mugo, B.W., Limbe, A.E. and Vulimu, E.M. (2005) Extending post-harvest life of sweet pepper(capsicum annum 1.'californiawonder)' with modified atmosphere packaging and storage temperature. Agriculture Tropicaetsubtropica, 38 (2), 28-34.

Porat, R., Pavoncello, D., Peretz, Y., Weiss, B., Cohen, L., Ben-Yehoshua, S., Fallik, E., Droby, S. and Lurie, S, (2000) Induction of resistance against Penicilliumdigitatum and chilling water brushing treatment. J. Hortic. Sci. Biotechnol., 75, 428-432.

Raymond, L.V., Zhang, M. and Roknul Azam, S.M. (2012) Effect of chitosan coating on physical and microbical characteristics of fresh-cut green peppers (Capsicum annum L.) Pakistan Journal of Nutrition, 11 (10), 806-811.

Reddy, B.M.V., Corcuff, R., Kasaai, M.R., Castaigne, F. and Arul, J. (1999) Induction of resistance againt gray mold rot in carrot root by chitosan. Phytopathology, 98, S6.

Risse, R.A. and Miller, W.R. (1986) Individual film wrapping of Florida cucumbers, eggplant, peppers and tomatoes for extending shelf life. J. Plastic Film and Sheeting, 2, 164-171. 
Rodriguez, S., Casolibe, R.M., Questa, A.G. and Felker, P. (2005) Hot water to reduce chilling injury and fungal development and improve visual quality of two Opuntiaficusindica fruit clones. Journal of Arid Enviroment, 63, 366-378.

Romanazzi, G., Nigro, Ippolito, A., Di Venere, D. and Salerno, M. (2002) Effects of pre-and postharvest chitosan treatments to control storage grey mould of table grapes. J. Food Sci., 67, 1862-1867.

Sabehat, A., Welss, D. and Lurie, S. (1996) The Correlation between heat shock protein accumulation and persistence and chilling tolerance in tomato fruits. Plant Physiol., 110, 531-537.

Sakaldaş, M. and Kaynaş, K. (2010) Biochemical and quality parameters changes of green sweet bell peppers as affected by different postharvest treatments. Journal of Biotechnology, 9 (48), 8174-8181.

Sala, J.M. and Lafuente, M.T. (2000) Catalase enzyme activity is related to tolerance of mandarin fruit to chilling. Postharvest Biology and Technology, 20, 81-89.

Sapitnitskaya, M., Maul, P., McCollum, G.T., Guy, C.L., Weiss, B., Samach, A. and Porat, R. (2006) Postharvest heat and conditioning treatments activate different molecular responses and reduce chilling injuries in grapefruit. J. Exp. Bot., 57, 2943-2953.

Shehata, S.A., Behairy, A.G. and Fawzy, Z.F. (2004) Effect of some organic manures on growth and chemical composition of sweet pepper (Capsicum annuumL.) grown in a sandy soil. Egypt. J. Agric. Res., 82 (2), 57-71.

Shehata, S.A., Hashem, M.Y., Emam, M.S. and Rageh, M.A. (2009) Effect of hot water and calcium chloride treatments on fresh cut sweet pepper during cold storage. Annals of Agric. Sci., Moshtohor, 47 (3), 445-455.

Snedecor, G.W. and Cochran, W.G. (1980).Statistical Methods. $8^{\text {th }}$ Ed., Iowa State Univ. Press, Ames, Iowa, USA., 476 p.

Vicente, A.R.,Matrtinez,G.A.,Chaves, A.R. and Civello, P.M. (2003) Quality of heattreated strawberry fruit during refrigerated storage. Postharvest Biology and Technology, 25 (1), 59-71.

Wang, C.Y. and Qi, L.L. (1997) Modified atmosphere package alleviates chilling injury in cucumber. Postharvest Biology and Technology, 10, 195-200.

Watada, A.E. and Qi, L. (1999) Quality of fresh-cut produce. Postharvest Biology and Technology, 15, 201-205.

Wilson, C.L., El-Ghaouth, A., Chaluts, E., Droby, S., Stevens, C., Lu, J.L., Khan, V. and Arul, J. (1994) Potential of induced resistance to control Postharvest diseases of fruits and vegetables. Plant Dis., 78, 837-844.

Xing, Y.G., Li, X.H., Xu, Q.L., Yung, J., Lu, Y.Q. and Tang, Y. (2011) Effect of chitosan coating enriched with cinnamon oil on qualitative properties of sweet pepper (Capsicum annum L.), Food Chem., 124,1443-1450.

Egypt. J. Hort. Vol. 42, No.1 (2015) 
Yonemoto Y. and Higuchi, H. K. (2002) Effect of storage temperature and coating on ethylene production, respiration and Shelf-Life in cherimoya fruit-Japanese soc for Hortsci., 71, 643-650.

Zhang, J., Huang, W., Pan, Q. and Liu, Y. (2005) Improvement of chilling tolerance and accumulation of heat shock proteins in grape berries (Vitisvinifera cv. Jingxiu)

\section{المحافظة على جودة ثمار القلقل الحلو: تأثير الماء الساخن

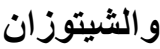
نعمة محمد حسين"، ممدوحِ محمد فوزى عبد الله *"، أحمد أبو اليزيد عبد الحافظ*"

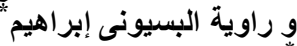

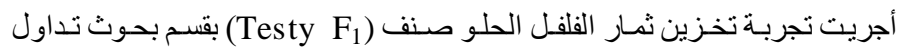

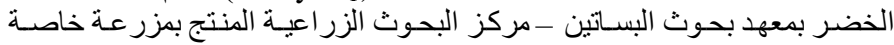

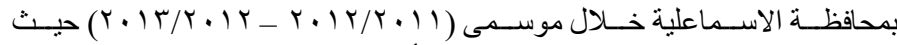

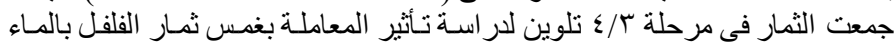

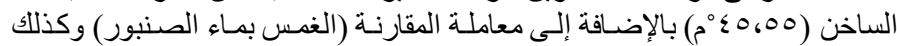

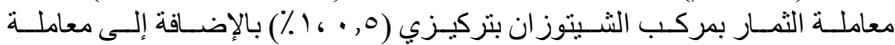

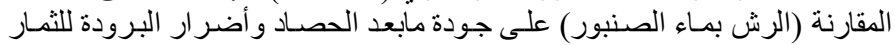

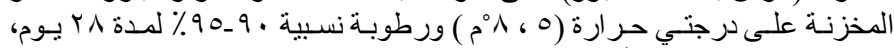

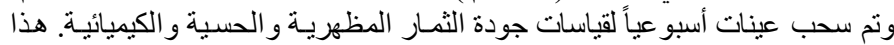

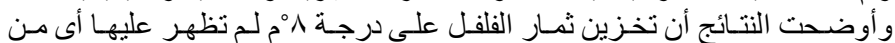

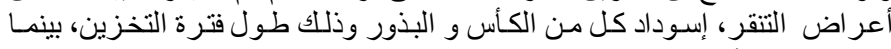

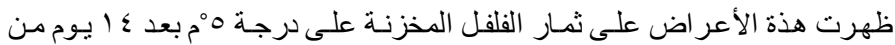

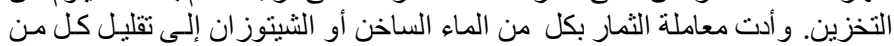

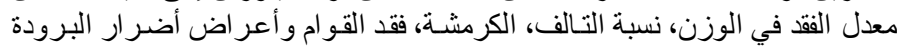

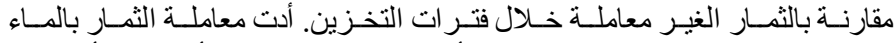

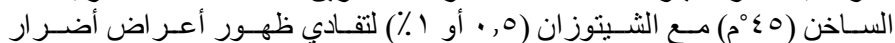

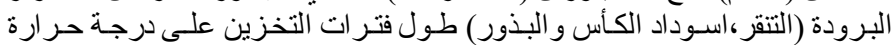

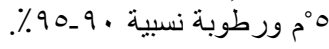

